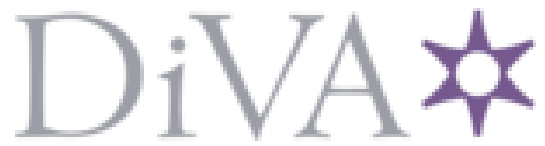

http://www.diva-portal.org

\title{
Postprint
}

This is the accepted version of a paper published in Synthese. This paper has been peerreviewed but does not include the final publisher proof-corrections or journal pagination.

Citation for the original published paper (version of record):

Pagin, P. (2017)

Tolerance and higher-order vagueness

Synthese, 194(10): 3727-3760

https://doi.org/10.1007/s11229-015-0798-x

Access to the published version may require subscription.

N.B. When citing this work, cite the original published paper.

Permanent link to this version:

http://urn.kb.se/resolve?urn=urn:nbn:se:su:diva-149870 
Published in Synthese 194:3727-3760

\title{
Tolerance and Higher-Order Vagueness*
}

\author{
Peter Pagin
}

April 23, 2018, 11:57

\begin{abstract}
The idea of higher-order vagueness is usually associated with conceptions of vagueness that focus on the existence of borderline cases. What sense can be made of it within a conception of vagueness that focuses on tolerance instead? A proposal is offered here. It involves understanding 'definitely' not as a sentence operator but as a predicate modifier, and more precisely as an intensifier, that is, an operator that shifts the predicate extension along a scale. This idea is combined with the author's earlier approach to the semantics of vague expressions, which builds on the idea of a central gap associated with a predicate. The central gap approach is generalized to handle arbitrarily many iterations of 'definitely'.
\end{abstract}

\section{Higher-order vagueness}

Although there is no uncontroversial way of characterizing vagueness (see e.g. Keefe and Smith 1996a Eklund 2007), two properties stand out as intuitively associated with typically vague predicates: that they are tolerant and that they have borderline cases. ${ }^{1}$

\footnotetext{
${ }^{*}$ I am much indebted too comments from two anonymous referees, and from Richard Dietz, which all helped improve the paper a lot, catching an error I had made in comparing the definitions of Fine and Williamson. I also benefited from comments at the Tokyo conference, in particular by Nicholas Smith and Wlodek Rabinowitz.

Versions of the talk have been presented at the LMPS congress in Beijing in 2007, at avagueness seminar at Institut Jean-Nicod, Paris, in 2010, at a vagueness conference in Amsterdam in 2012, at the Vagueness and Probability Conference in Tokyo in 2013, and at the department of philosophy, Stockholm University, in March 2015. I am grateful to comments from many persons at those occasions, and there is not room to list all. Still, I'd like to mention in particular Jerry Seligman, Paul Egré, Denis Bonnay, Dave Ripley, Elia Zardini, Robert van Rooij, Chris Barker, Pablo Cobreros, Agustin Rayo, Jonas Åkerman, Dag Prawitz, Per Martin-Löf, Dag Westerståhl, Valentin Goranko, Anandi Hattiangadi, Åsa Wikforss, and Kathrin Glüer-Pagin.

Work on this paper was supported by a grant from the Swedish Research Council (VR), for the project Vagueness and Context Factors.

${ }^{1}$ Two others that are also known: that vague predicates are sorites-susceptible, and that they have no sharp boundaries. The former is a consequence of tolerance. The latter may be seen as resulting from tolerance and the existence of borderline cases together.
} 
That a predicate is tolerant means roughly that it is insensitive to small differences ${ }^{2}$ This lack of sensitivity can be expressed by so-called tolerance principles. For a vague predicate such as 'tall', assuming that it is insensitive to differences of $1 \mathrm{~mm}$, it could be expressed as follows

(1) If a man of $n+1 \mathrm{~mm}$ is tall, then a man of $n \mathrm{~mm}$ is tall.

Under an intuitive interpretation, [1], together with premises such that a man of 2000 $\mathrm{mm}$ is tall and a man of $1600 \mathrm{~mm}$ is not tall, yields a contradiction. Applying (1), step by step, from the clear positive case $(2000 \mathrm{~mm})$, we reach the conclusion that a man of $1600 \mathrm{~mm}$ is tall as well, contradicting the intuitive verdict that such a man is not tall. This is the sorites paradox. Because tolerance, taken literally as semantic insensitivity to small difference, tends to generate the sorites, most accounts of vagueness take tolerance principles such as (1) to be false, or at least not true.

To be borderline something is, roughly, to be not uncontroversially that thing, nor uncontroversially not that thing. Typically, this is expressed by means the adverbs 'clearly' or 'definitely'. To be borderline tall is then to be neither definitely tall nor definitely not tall. On some accounts, like supervaluationism (Fine 1975, Keefe 2000), a borderline case for 'tall' is a man for which neither the predicate nor its negation applies. That is, if John is borderline tall, then the sentence 'John is tall' is neither true nor false. This is a semantic conception of borderline status. According to Stephen Schiffer (2003), the sentence is strictly indeterminate in truth value, and there is a special kind of belief that concerns vagueness. For epistemicism (cf. Williamson 1994, Sorensen 1988), vague predicates do not induce indeterminacy, nor lack of truth value. Borderline status is not a semantic status at all, but an epistemic status. If John is borderline tall, we cannot know whether he is tall. On Williamson's explanation, knowledge requires a margin of error, and for borderline cases the required margin is lacking.

Even if conceptions of borderline and of definiteness differ, their internal relation is generally accepted. Given the possibility of borderline cases of tallness, we might divide (relative to some contextual standard) people into definitely tall, borderline, and definitely not tall. The idea of higher-order vagueness relates to this possibility: there might be borderline cases between the definitely tall and the borderline, as well as between the borderline and the definitely not tall. This would mean that 'definitely tall' as well as 'borderline tall' and 'definitely not tall' are all vague, but also that 'tall' itself has secondorder vagueness. The idea of second-order vagueness, as well as the idea that ordinary vagueness inescapably involves second-order vagueness as well, is clearly presented in

\footnotetext{
${ }^{2}$ The conception of tolerance is due to Crispin Wright $(1975 ; 1976)$.
} 
Russell (1923), where 'the penumbra' is his term for the borderline area:

Someone might seek to obtain precision in the use of words by saying that no word is to be applied in the penumbra, but unfortunately the penumbra itself is not accurately definable, and all the vagueness which apply to the primary uses of words apply also when we try to fix a limit to their indubitable applicability (Russell 1923 63-4.)

Once the idea of second-order vagueness is accepted, it is natural to express it by iterating 'definitely': we distinguish between the definitely definitely tall, the borderline definitely tall, and the definitely not definitely tall ${ }^{3}$ Since iteration can be repeated, the idea of higher-order vagueness is close at hand $4^{4}$ The idea that there can always be a borderline case between being borderline and being not borderline, i.e. there is never a sharp boundary between borderline and not borderline, is well characterized by saying that the boundary of a predicate is blurred, or fuzzy $5^{5}$

It has become standard to formalize 'definitely' as a sentence operator $D($ or $\Delta)$. Then, rather than characterizing objects as being borderline cases of a predicate or a property, sentences are borderline or not. So

$$
\neg D p \& \neg D \neg p
$$

is true just in case $\ulcorner p\urcorner$ is a borderline sentence (I shall in the remainder be less careful with the quote marks). I don't find it intuitively clear how to understand $D$ as a sentence operator that can take any sentence as argument. We can well understand an instance of (2) where $p$ is replaced by simple atomic sentence:

$$
\neg D F t \& \neg D \neg F t
$$

\footnotetext{
${ }^{3}$ Second-order vagueness is not universally accepted. Crispin Wright in several papers (e.g. 2010) and Diana Raffman (2010), as well as Suzanne Bobzien (e.g. 2010), are critical of the idea of applying 'definitely' to borderline. For instance, Raffman $(2010,513)$ argues that if something is definitely $\Phi$, then failure to classify it as $\Phi$ is mistaken or improper, while classifying something as borderline $\Phi$ is always optional, and then judging something to be definitely borderline $\Phi$ is inconsistent: it must then both be optional and mistaken to judge it to be $\Phi$.

However, what is optional about calling something borderline $\Phi$ is that it might also be called $\Phi$ as well as not $\Phi$, not that it might also be classified as not borderline $\Phi$. Should something be borderline borderline, then it should be optional as well to classify it as not borderline, but exactly this is ruled out if it is definitely borderline. There is no inconsistency here, as far as I can see.

${ }^{4} \mathrm{An}$ alternative idea of higher-order vagueness concerns vagueness in the meta-language, e.g. in a metalanguage that contains a truth predicate. I shall not be concerned with that idea here.

${ }^{5}$ This conception, attributed to Russell in the quoted passage, is sometimes called Radical Higher-order Vagueness. The idea is critically scrutinized in a recent paper by Richard Dietz [2015). Discussion of his points must be deferred to another occasion.
} 
for this can be translated into saying that the referent of $t$ is neither definitely $F$ nor definitely not $F$. But there is no simple translation when $p$ is e.g. a disjunction, or some quantified sentence.

Zardini (2013, p. 29) summarizes (without endorsement) some options proposed in the literature for defining $D$ as a sentence operator:

Semantic: $\quad$ Speakers' practices determine a sufficient truth-conditions for ' $\phi$ ', and the condition is satisfied.

Ontic: $\quad$ It is a fact of the matter that $\phi$.

Epistemic: $\quad$ No obstacle of a certain kind prevents knowledge that $\phi$.

Psychological: It is correct to hold a standard (possibly partial) belief to the effect that $\phi$.

(see op cit. for further references.) These ideas are very general in character and further claims and explanations are needed to connect them with vagueness in particular, and to block unwanted combinations with other sources than vagueness of loss of truth conditions, facthood, knowledge, or belief correctness. I shall not pursue the issue here.

One way to look at it formally, which fits possible-worlds frameworks that are often employed, is to take sentences to express properties of possible worlds. That sentence $p$ is borderline can then be understood as saying that the actual world is a borderline case of the property $\llbracket p \rrbracket$ (where $\llbracket \rrbracket$ is the semantic function), or similarly that the actual world is a borderline case of the truth of $p$. This fits the approaches of e.g. Fine and Williamson (see below) ${ }^{6}$

Williamson $(1999,131)$, in a model-theoretic treatment, defines a formula $\phi$ to be precise just in case

$$
\vDash D \phi \vee D \neg \phi
$$

and glosses this by saying that "it is semantically guaranteed that it is definite whether $\phi$ holds" ${ }^{7}$ He then adds that $\phi$ is vague just in case it is not precise. There are corresponding concepts of being precise [vague] in a model $M$.

First-order vagueness considers two boundaries (between definitely $F$ and not definitely $F$, and between definitely not- $F$ and not definitely not- $F$ ), second-order vagueness four boundaries (between definitely definitely $F$ and not definitely definitely $F$, etc.), and

\footnotetext{
${ }^{6}$ As we will see later on, this is not a general recipe, for it does not tell us how to aggregate the status of different individuals with respect to different predicates into the status of a world with respect to a sentence.

${ }^{7}$ He uses ' $\Delta$ ' where I use ' $D$ '. My choice is merely aesthetic.
} 
$n$ :th order vagueness $2^{n}$ boundaries, and on Williamson's definition, $\phi$ is $n$ :th order precise only if all the $2^{n}$ boundaries are sharp, i.e. only if all the $2^{n}$ combinations of negation and $n$ occurrences of $D$ are precise. ${ }^{8}$

This idea fits well a conception of vagueness as having borderlines. A particular formula is then $n$ :th order vague just in case it is located on some $n$ :th order borderline, and is otherwise $n$ :th order precise. But under a conception of vagueness as tolerance, vagueness consists in weakness of discrimination in general between similar items. So on a tolerance conception, vagueness is not a property only of borderline sentences.

It is true that tolerance is harmless except in borderline areas. It is also true that under certain conditions, with non-empty extension and anti-extension of the predicate, and certain distributions of individuals in the domain, borderline cases will result. Still, given a semantics that validates tolerance principles, borderline cases will give rise to a sorites contradiction, and hence the admissibility of borderline cases must be very much restricted. Under these circumstances, what room is there for higher-order vagueness?

There is room for a closely related concept, formally similar, given that there is an interpretation of 'definitely' in a tolerance-friendly semantic framework. There are two characteristic properties of definiteness that can be implemented in such a framework:

(MoreSafe) Something that is definitely $F$ is at a safer distance, or at worst a no less safe distance, from being not- $F$ than what is required of simply being $F$.

(LessTolerant) The tolerance of 'definitely $F$ ' is lower than, or at worst as low as, the tolerance of $F$ alone: the distance required between being $F$ and being not- $F$ is greater than, or equal, to the distance required between being definitely $F$ and not being definitely $F$.

These two properties can be implemented separately or jointly. We shall see how in section 6 . Before that, there are a few things to rehearse, to begin with, the normal interpretations of definitely proposed by Fine and Williamson.

\footnotetext{
${ }^{8}$ Cf. Sainsbury 1990 p. 255; 1991 p. 169. Williamson 1999 p. 135), using the predicate example 'is such that 7 is small', criticises the idea that a predicate for which every case is borderline draws more boundaries than does a predicate for which no case is borderline. I find it hard to see the relevance of the objection, however, since a boundary is drawn to the extent that the condition to be satisfied on the one side is different from the condition to be satisfied on the other side, regardless of how many objects, if any, satisfy them.

The question when we are justified in distinguishing the conditions is more difficult. It is plausible to say that if the predicates $F$ and $G$ are logically equivalent (e.g. if $\forall x(F x \leftrightarrow G x)$ is logically true), then the condition of satisfying the one just is the same as the condition of satisfying the other.
} 


\section{Normal interpretations of $D$}

In the supervaluationist framework of Fine 1975 pp. 293-4, the $D$ operator is defined by means of sequences of nested sets of specifications. A complete specification is (or corresponds to) a complete assignment of truth values to atomic sentences. If the language has first-order vagueness, there is no uniquely admissible complete specification, but a set of admissible ones. If the language has second-order vagueness, there is no unique admissible set of admissible complete specifications, but a set of admissible sets. If there is third-order vagueness, there is a set of admissible sets of admissible sets, and so on.

Formally, Fine defines a zero-th-order space as a complete specification, and an $n+1$ order space as a set of $n$ :th order spaces. An $n$ :th order boundary is a sequence $s^{0}, s^{1}, \ldots, s^{n}$ such that $s^{i}$ is an $i$ :th order space, $i \leq n$, and $s^{j} \in s^{j+1}, j<n$; an $\omega$-order boundary, or simply boundary, is an infinite sequence $s^{0}, s^{1}, \ldots$ such that each $s^{i}$ is an $i$ :th order boundary, $i=1,2 \ldots$. A boundary is admissible iff each of its terms is.

A relation $R$ between boundaries is such that, where $b=b_{1}, b_{2}, \ldots, c=c_{1}, c_{2}, \ldots$,

$$
b R c \quad \text { iff } \quad c_{i} \in b_{i+1}, i=1,2 \ldots
$$

It is immediate that if $b$ is admissible, and $b R c$, then $c$ is admissible, and also that $R$ is reflexive.

Then Fine gives the clause for $D$ :

(D-F) $\quad b=D \phi \quad$ iff $\quad$ for all boundaries $c, b R c$ iff $c=\phi$.

On this semantics, $D$ behaves like a necessity operator, with boundaries as worlds, and $R$ as an accessibility relation. According to Fine, the resulting modal logic is T.

Fine $(1975,287)$ also defines indefiniteness of a sentence, corresponding to Williamson's vagueness of a formula, somewhat differently:

$$
\begin{aligned}
& \text { (H-F) i) } I^{0} \phi=\phi \\
& \text { ii) } I^{n+1} \phi=\neg D I^{n} \phi \& \neg D \neg I^{n} \phi \\
& \text { iii) What } a \text { denotes is an } n \text { :th order borderline case of } F \text { iff } I^{n} F a \text { is true }{ }^{9}
\end{aligned}
$$

In Williamson 1999, $D$ is also taken as a necessity-like operator. His preferred epistemicist interpretation of $D$ (Williamson 1999, 128) is such that definiteness consists in truth under all interpretations of the language that are indiscriminable from the right one (the interpretation the language actually has as used by its speakers). Each possible world, or point of evaluation, is then an interpretation of the language, and the relation

\footnotetext{
${ }^{9}$ I have followed Williamson in using the inductive format.
} 
of indiscriminability is the accessibility relation between worlds.

In Williamson's model theory, a model $M$ is a triple $\langle W, R, \llbracket \cdot \rrbracket\rangle$, where $W$ is a set of points (worlds), $R$ a binary relation on $W$ (accessibility) and $\llbracket \cdot \rrbracket$ an interpretation function, a mapping from formulas to sets of points (where the formula is true). The interpretation of a conjunction is the intersection of the interpretation of the conjuncts, and the interpretation of a negation is the complement in $W$ of the interpretation of what is negated. The clause for the $D$ operator is

$(\mathrm{D}-\mathrm{W}) \llbracket D \phi \rrbracket=\{w \in W: \forall x \in W(w R x \Rightarrow x \in \llbracket \phi \rrbracket)\}$

In the algebraic format, this is just the standard necessity clause $(D \phi$ is true at a world $w$ just in case $\phi$ is true is all worlds accessible (indiscriminable) from $w$ ). As stated by Williamson, the semantics validates the modal logic B (or KTB), given by the following axioms and rules:

$$
\begin{array}{ll}
\text { PC } & \text { If } \phi \text { is a truth-functional tautology, then } \vDash \phi \\
\mathrm{K} & =D(\phi \rightarrow \psi) \rightarrow(D \phi \rightarrow D \psi) \\
\mathrm{T} & =D \phi \rightarrow \phi \\
\mathrm{B} & =\phi \rightarrow D \neg D \neg \phi \\
\mathrm{MP} & \text { If } \vDash \phi \rightarrow \psi \text { and } \vDash \phi, \text { then } \vDash \psi \\
\mathrm{RN} & \text { If } \vDash \phi, \text { then } \models D \phi
\end{array}
$$

The B schema is not valid on Fine's semantics (Fine 1975, pp. 293-4; Fine's accessibility relation is not symmetric). Removing it results in the modal logic T, the logic of $D$ according to Fine's semantics 10

Williamson $(1999,132)$ formally defines higher-order vagueness by means of syntactically defined sets of sentences: Given a set $\mathrm{X}$ of sentences, $\mathbb{C X}$ is the closure of $\mathrm{X}$ under truth-functional operators. Such a set is called a classification by Williamson. A classification is precise just in case all its members are precise, in the sense of (4). Next, he adds an inductive definition, and a definition of higher-order vagueness:

$(\mathrm{H}-\mathrm{W})$ i) $\quad \mathbb{C}_{1} \mathrm{X}=\mathbb{C X}$

ii) $\mathbb{C}_{n+1} \mathrm{X}=\mathbb{C}\left\{D \phi: \phi \in \mathbb{C}_{n} \mathrm{X}\right\}$

iii) $\phi$ is $n$ :th order precise iff $\mathbb{C}_{n}\{\phi\}$ is precise and $\phi$ is $n$ :th order vague iff $\phi$ is not $n$ :th order precise.

By this definition, a formula $\phi$ is second-order vague just in case some formula in $\mathbb{C}\{D \psi$ :

\footnotetext{
${ }^{10}$ It should be noted that Williamson is not wholly committed to the validity of B, but discusses reasons for abandoning it (Williamson 1999 pp. 137-8). Still, he does assume it.
} 
$\psi \in \mathbb{C}\{\phi\}\}$ is vague. So for Williamson, it is sufficient for $\phi$ to be second-order vague if $D \phi$ is vague, i.e. if true:

$$
\neg D D \phi \wedge \neg D \neg D \phi
$$

This is compatible with $D \neg \phi$ not being vague, and hence with the falsity of

$$
\neg D D \neg \phi \wedge \neg D \neg D \neg \phi
$$

This is in fact mandated by the B axiom. For if $\phi$ is true, the B Axiom is inconsistent with the truth of the second conjunct of 6 , while if $\phi$ is false, it is inconsistent with the truth of the second conjunct of (5). So, in KTB, at most one of the two can be true.

Intuitively, however, if $\phi$ is vague, and higher-order vagueness is admitted, then both $D \phi$ and $D \neg \phi$ are vague, or at least can be, on any view that minimally approximates Russell's. That $D \neg \phi$ is vague should intuitively amount either to the tolerance of the sentence as expressing a predicate of worlds, or amount to the existence of a borderline case, i.e. the existence of a world $w$ such that (6) is true with respect to $w$, i.e. of a world that is neither $D D \neg \phi$ nor $D \neg D \neg \phi$. That would not have to be the actual world, only some world or other. However, when using $D$ as necessity-like sentence operator to characterize vagueness the way it is done by Williamson or Fine, all sentences used (when unembedded) provide properties of exactly the actual world, insofar as they are only evaluated for simple truth or falsity. The same world cannot intuitively be both borderline $D \phi$ and borderline $D \neg \phi$, but the fact that both properties can be instantiated, by different worlds, indicates that truth of modal sentences at the actual world might not be the most adequate way to characterize higher-order vagueness. One can get around this limitation by explicitly considering the truth of sentences relative to worlds (as is sometimes done). Formally, however, this is equivalent to treating sentences a predicates of worlds, and $D$ as an operator on such predicates.

Williamson's choice of definition is crucial to his central claim that second-order vagueness entails higher-order vagueness of all finite orders (see below, section 8). There, it is assumed that we have both first-order and second-order vagueness. This corresponds here to the idea that the actual world is borderline $\phi$ as well as borderline $D \phi$, which itself again is fairly unintuitive on a scale-based approach (applied to worlds).

Both Fine's and Williamson's semantics validate the K schema (the closure of $D$ under the conditional), as well as the RN rule (corresponding to the rule necessitation in standard modal logic). Their $D$ logics are therefore normal modal logics. In the semantics for $D$ to be presented below, different structural properties will result from different settings of the two crucial parameters, relating to MoreSafe and to (LessTolerant). 
Before setting out the semantics for $D$ as a tolerance operator, I shall give a brief presentation of the semantic framework of the central gap account of vagueness ${ }^{11}$

\section{Gap semantics for vague predicates}

Gap semantics is intended to provide an interpretation of vague, i.e. tolerant, vocabulary, in normal usage ${ }^{12}$ It is not intended to solve the sorites paradox, but rather to offer an account of how the paradox can be avoided in most contexts. It is based on the intuition that when speakers apply vague predicates to contextually clear cases, they act as if there are only clear cases, i.e. leave borderline, or problematic, cases out of consideration. This is formally modeled as if there is a central gap in the scale associated with a predicate $F$, such that no individual in the relevant domain has a value in that part of the scale. The gap in the scale must exceed or at least match the tolerance level. For instance, assuming a tolerance of $1 \mathrm{~mm}$ for 'tall' as applied to adult men, we can have a central gap for 'tall' between $180 \mathrm{~cm}$ and $170 \mathrm{~cm}$. Everyone with a height above the gap is tall, and everyone with a height below the gap is not. In such a situation, a tolerance principle such as

For every $x$ and $y$, if $x$ has height at most $k+1 \mathrm{~mm}$ and $y$ has height $k \mathrm{~mm}$, then if $x$ is tall, so is $y$

given a domain of quantification involving just adult males, say, is true. It does not give rise to paradox in that domain, since the domain does not contain a sorites sequence for 'tall'.

The main idea of Gap Semantics is that predicates are interpreted together with a contextually given quantifier domain restriction, such that after the restriction is applied, the domain has a central gap. This is done for each vague predicate used in the context. Technically, it will be convenient to say that a domain has a central gap even when it is not "central", i.e. in case everything is $F$ or everything is not $F$. If the domain has a central $F$-gap (whether or not central), we say that then the domain is F-relaxed.

The semantics has the following properties: the interpretation function $\llbracket \cdot \rrbracket$ is induced by a gap function $\mathscr{G} . \mathscr{G}$ is defined for a predicate $F$ in a domain $E$ iff $E$ is $F$-relaxed or allows a restriction that makes it $F$-relaxed. When this condition is met for a context $c$, the interpretation of speakers in $c$ gives a classical (bivalent) semantics where a suitable tolerance principle is true.

It will be assumed that in each context $c$, for each vague predicate $F$ that is used in

\footnotetext{
${ }^{11}$ Fara 2002 p. 136) in fact provides a model with a central gap in a domain of possible worlds, relative to a distance measure between worlds. She calls models that lack such a gap "stepping-stone" models.

${ }^{12}$ This account is presented and elaborated in Pagin 2010 Pagin 2011b Much of the material in this section is taken from the latter.
} 
$c$, a central gap is determined for $F$. This is an idealization. In any actual occasion of speech, it will be left undetermined exactly what the tolerance level is and where the gap is. It would be more realistic to speak of what is admissible on a certain occasion. This is all the more realistic since the contextual parameters will have to depend to some extent on what is uttered on the occasion.

It will be required that a full semantics is given in each context for the full fragment of a language that is used in the context, but not for linguistic material outside that fragment. This means in particular that any referring singular term that is used in $c$ must have a referent in the quantifier domain of $c$. For maintaining bivalence, the relevant measure of that term for the predicate $F$ cannot fall in the central gap for $F$ at $c$. Hence, the central gap is required not to include the measure of that referent. So the location of the gap depends on the terms used in the context. This will be a requirement on any admissible gap function $\mathscr{G}$. I shall operate with just one such function.

This function must to some extent be arbitrary (a choice function), but must also be subject to a number of restrictions:

(GAP) i) The central gap must be selected so that the full fragment of language used in the context, including pragmatically determined contextual updates, is taken account of.

ii) The size of the central gap must be at least equal to the tolerance level.

iii) The location of the central gap must be selected so as to ensure consistency.

iv) The location of the central gap must selected so that what the speaker says comes out as true, to the extent this is possible and reasonable.

The order of these selection principles is meant to reflect the order of importance. Indeed, GAPi) cannot be compromised, but this has the consequence that the other three may not be jointly satisfiable, given collateral facts.

It is psychologically plausible that the gap is greater, and even considerably greater, than the tolerance level, but for the purpose of setting out the semantics, it is more elegant to reduce the two contextual parameters, tolerance level and gap size, to a single parameter. We can let the gap size equal the tolerance level. Then, the requirement GAPii will be automatically met.

Here I shall follow the tradition (from Westerståhl 1985 and onwards) in representing a contextually determined domain restriction as an intersection with the first argument of the determiner denotation. In order to implement the domain restriction of the central gap account, there will be two further restrictions, assuming that both the first and the second argument of the determiner is vague. In a particular context $c$, a central gap 


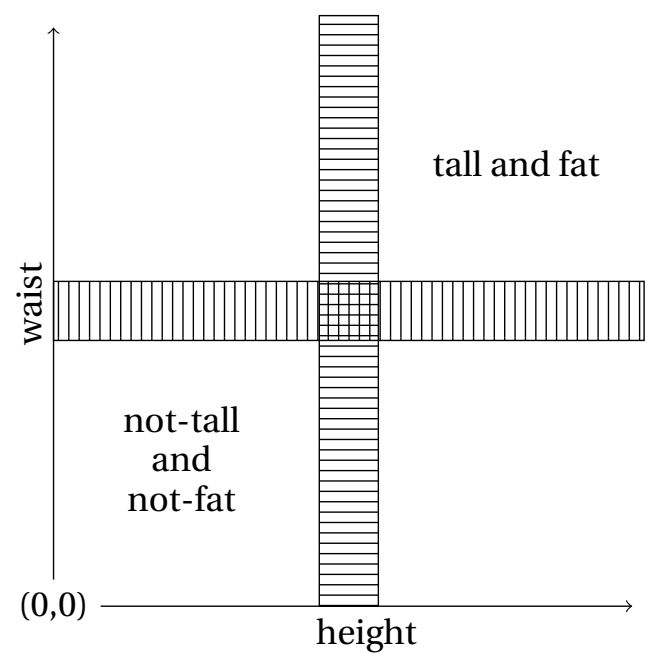

Figure 1: Union of domain cuts.

will be associated with each predicate in the lexicon that has been used in the context. Let us assume that each vague lexical item is associated with a measure function. Where $F$ is a predicate, let $\nabla(c, F)$ be the $F$ domain cut, the set of objects whose measures are within the central gap associated with $F$ in $c$. We take it to return the empty set in case the predicate in question is not vague. Let $\nabla(c, \bar{F})$ be

$$
\bigcup_{i=1}^{n} \nabla\left(c, F_{i}\right)
$$

the union of domain cuts for predicates $F_{i}, \ldots, F_{n}$ in context $c$. For two atomic predicates used together, the union is represented in Figure 1 as comprising two intersecting bands in the plane ${ }^{13}$ In the restricted domain, no object has a pair of measures in either band. With respect to

\section{Most men run}

we will then have a domain that contains (at most) man $-(\nabla(c$, man $) \cup \nabla(c$, runs $))$.

We assume a basic domain $E$ of individuals, and a domain $C$ of contexts. We shall let each atomic $n$-place predicate, for $n \geq 1$, be associated with an $n+1$-place function from $E^{n} \times C$ to the set of truth values $\{0,1\}$. However, the vagueness of many-place predicates has not been much discussed in the literature, and I too shall restrict the discussion to

\footnotetext{
${ }^{13}$ This can also be regarded as the two-dimensional gap for complex predicates such as 'is tall and fat' and 'is tall or fat'. Cf. the final section.
} 
one-place predicates, among the atomic ones.

We assume a language $L$ with a finite stock $L_{A}$ of atomic one-place predicates. We also assume a classical language with negation, conjunction, and universal quantification as primitive, and the others classically defined as usual. We also have a set $L^{*}$ of fragments of $L$, where each fragment $l \in L^{*}$ has the same basic vocabulary as $L$ except for containing only a subset of the set of closed singular terms of $L$ and a subset of the set of vague atomic one-place predicates of $L$. Each fragment is closed under the same syntactic operations as $L$ itself. We further have a fragment function $\mathscr{F}: C \longrightarrow L^{*}$ from contexts to fragments of $L$. Let $\mathscr{F}_{0}(c)$ be the set of atomic one-place predicates in $\mathscr{F}(c)$ and $s^{0}$ be the set of one-place predicates in the sentence $s$. Then we shall require that

(FP) If $s$ is used in context $c$, then $s^{0} \subseteq \mathscr{F}_{0}(c)$

With each atomic predicate $F_{i} \in L_{A}$ is associated a weak order $\lesssim F_{i}$ (a dimension of variation) and an admissible (at least interval scale) measure function $\mathscr{H}_{F_{i}}: E \longrightarrow \mathbb{R}$ (a function from individuals to real numbers, relative to a scale) ${ }^{14}$ We further have a function $\mathscr{T}_{c}(F)$ that for a context $c$ and an atomic predicate $F$ returns a tolerance level for $F$ at $c$. We also have a central gap function $\mathscr{G}_{c}(F)$ from a predicate $F$ and a context $c$ to pairs $(i, j)$ of real numbers, $i \geq j$, with respect to the measure function $\mathscr{H}_{F}$. If $F$ is vague, then $i>j$. We shall refer to the left member as $\mathscr{G}_{c}^{+}(F)$ and to the right member as $\mathscr{G}_{c}^{-}(F)$.

To maintain consistency we need the condition that:

(GT) For every predicate $F \in L$ and context $c \in C$ it holds that

$$
\mathscr{G}_{c}^{+}(F)-\mathscr{G}_{c}^{-}(F) \geq \mathscr{T}_{c}(F)
$$

That is, the central gap of the context must be at least as great as the tolerance level of the context. As already suggested, however, it will be convenient to let the size of the central gap equal the tolerance level, since then an increase of precision, i.e. a reduction of the tolerance level, automatically reduces the central gap. Hence, we stipulate that:

$\left(\mathrm{GT}^{=}\right)$For every predicate $F \in L$ and context $c \in C$ it holds that

$$
\mathscr{G}_{c}^{+}(F)-\mathscr{G}_{c}^{-}(F)=\mathscr{T}_{c}(F) \text {. }
$$

When several atomic predicates are used in the same context $c \in C$, it will be required that individual terms do not have referents with measures in any of the gaps associated

\footnotetext{
${ }^{14}$ A weak order $\lesssim$ over a domain $D$ is a binary relation over $D$ satisfying the conditions

i) For all $d, d^{\prime} \in D, d \lesssim d^{\prime}$ or $d^{\prime} \lesssim d \quad$ (connectedness)

ii) For $d, d^{\prime}, d^{\prime \prime} \in D$, if $d \lesssim d^{\prime}$ and $d^{\prime} \lesssim d^{\prime \prime}$, then $d \lesssim d^{\prime \prime} \quad$ (transitivity)
} 
with the predicates used in $c$, hence that they have referents outside $\nabla(c, \bar{F})$.

Let $f, f^{\prime}$ etc. be assignment functions from variables $x_{1}, x_{2}, \ldots$ in $L$ to objects in $E$. The semantic function $\llbracket \cdot \rrbracket$ maps a sentence $s$, a context $c$ and an assignment function $f$ onto a truth value in $\{0,1\}$. Let $\llbracket F \rrbracket_{c}^{+}=\left\{a: \mathscr{H}_{F}(a)>\mathscr{G}_{c}^{+}(F)\right\}$, and let $\llbracket F \rrbracket_{c}^{-}=\left\{a: \mathscr{H}_{F}(a)<\mathscr{G}_{c}^{-}(F)\right\}$. For a singular term $t$, let $\llbracket t \rrbracket_{f}$ be $\llbracket t \rrbracket$ if $t$ is a constant, and $f(t)$ if $t$ is a variable. The relation $f^{\prime}[x] f$ holds iff the assignment $f^{\prime}$ differs from $f$ at most in what it assigns to $x$. Then we have the truth definition:

(GS) i) Where $t$ is a constant, $\llbracket t \rrbracket \notin \nabla(c, \bar{F})$

ii) Where $F$ is an atomic one-place predicate,

$$
\llbracket F t \rrbracket_{f, c}=1 \text { iff } \llbracket t \rrbracket_{f} \in \llbracket F \rrbracket_{c}^{+}
$$

iii) $\llbracket \neg A \rrbracket_{f, c}=1$ iff $\llbracket A \rrbracket_{f, c}=0$

iv) $\llbracket A \& B \rrbracket_{f, c}=1$ iff $\llbracket A \rrbracket_{f, c}=1$ and $\llbracket B \rrbracket_{f, c}=1$

v) $\llbracket Q x(A x, B x) \rrbracket_{f, c}=1$ iff

$$
\llbracket Q \rrbracket f^{\prime}\left(f^{\prime}[x] f \text { and } \llbracket A x \rrbracket_{f^{\prime}, c}=1 \text { and } f^{\prime}(x) \notin \nabla(c, \bar{F}), \llbracket B x \rrbracket_{f^{\prime}, c}=1\right)
$$

According to $\mathrm{V}$, in a context $c$, a sentence $Q(A x, B x)$ is true just in case $\llbracket Q \rrbracket$ many individuals of which $A x$ is true in $c$ and that do not belong in the contextually determined cut $\nabla(c, \bar{F})$ are such that $B x$ is true of them as well.

We can verify that a tolerance principle (equivalent to the prenex normal form (7)) such as

$$
\begin{aligned}
& \text { Some } x(\operatorname{man}(x) \& \operatorname{height}(x) \leq n+k \mathrm{~mm}, \operatorname{tall}(x)) \quad \rightarrow \\
& \operatorname{All} x(\operatorname{man}(x) \& \operatorname{height}(x) \geq n \operatorname{mm}), \operatorname{tall}(x)
\end{aligned}
$$

is true in any context $c$ where the tolerance level for 'tall' is $k \mathrm{~mm}$ or greater. We assume that the relevant measure function maps men on their heights in mm. Let us assume here that 'man' is non-vague. Then the antecedent of (9) is true just in case some individual $a$ in the restricted domain is a man and has a height above the upper edge of the 'tall' gap in $c$. Then any individual $b$ in the $c$-restricted domain that is a man and has a height at least that of the height of $a$ minus $k \mathrm{~mm}$, itself has a height above the upper edge of the gap, for there is no individual in the restricted domain that has a height in the gap, and any individual $c$ in the domain with a height below the gap is more than $k \mathrm{~mm}$ shorter than $a$. So the the consequent of 9 is true.

\section{Das a tolerance operator}

The present treatment of $D$ as a tolerance operator will fit into the Gap Semantics. One main difference between this account and those of Fine and Williamson is that $D$ here 
will be a predicate operator (predicate modifier) rather than a sentence operator ${ }^{15}$ Where $F$ is a one-place predicate, $D F$ will again be a one-place predicate. I shall write ' $D F(t)$ ', meaning that the referent of $t$ is definitely $F{ }^{16} F$ itself can be simple or complex, and in principle we allow for all Boolean combinations. Thus we can use the form $D(F \& G)(t)$ to say things such as

\section{Bill is definitely both tall and bald}

However, in the present paper, only the semantics for simple predicates will be developed, since allowing for two-place connectives under $D$ gives rise to considerable complications. Despite this difference to more standard treatments of $D$, the semantics will to a large extent be analogous to a semantics for the sentence-operator counterpart. We can heuristically picture the predicates as feature-placing sentences that are true at worlds, where the worlds in question are the individuals the predicates are true or false of in the usual sense. Thus, tallness is "true at" John just in case John is tall. In such a modal treatment, $D$ is again given a necessity-like interpretation. Accordingly, we shall say that $D F$ is true at, or of, an individual $a$ just in case $F$ is true of every individual $b$ that stands in a certain relation $R$ to $a$. This relation $R$, the counterpart to the accessibility relation, will be associated with the central gap $\mathscr{G}_{c}(F)$ of $F$.

Two basic features of this approach deserve immediate highlight. First, the approach is scale-based, just as the basic gap semantics. Whether $D F$ is true of a particular object $a$ depends on the position of the measure of $a$ on the relevant scale, and in particular on its position in relation to the requirements for being $F$. The second basic feature is that the distance requirement is not fixed, but may both vary between worlds and in addition depend on $F$. If we regard the relation as a certain margin, the margin may depend on the predicate itself, as we shall see.

The main idea is that $D$ is interpreted by means of two functions on real numbers $\delta$ and $\epsilon$ that are associated with the ideas of reduced tolerance and added safety ${ }^{17}$ The function $\delta$ maps the contextual tolerance level of $F$ on the contextual tolerance level

\footnotetext{
${ }^{15}$ With respect to gradable adjectives, the present interpretation of 'definitely' takes it to be an intensifier, moving the application conditions along the scale, as does the interpretation of 'very'. This does not, of course, mean that I take 'definitely' to mean the same as 'very'.

${ }^{16}$ In the literature on higher-order vagueness it is common to treat the definitely operator formally as a sentence operator, but to revert predicate modifier talk informally: saying that an object $a$ definitely $F$ when it is formally a question of a sentence or proposition being definitely true.

${ }^{17}$ I have several times been asked why we need two parameters rather than just one. It is indeed possible to fix a relation between $\delta$ and $\epsilon$ so that one is a function of the other. However, I see no reason why any particular relation between $\delta$ and $\epsilon$ would be selected either by the meanings of predicate, the meaning of 'definitely', or the ideas of vagueness or higher-order vagueness. So fixing a relation would be arbitrary, and thus an unreasonable restriction on the domain of models.
} 
of $D F$, and $\epsilon$ maps the tolerance level of $F$ on the safety margin of $D F$ to $F$, i.e. the guaranteed distance to the "smallest" $F$ :s. This safety margin will also fix the upper edge of the gap of $D F$.

Assume that a gap function $\mathscr{G}$ is defined both for $F$ and for $D F$. Then we have:

$$
\begin{array}{ll}
\text { i) } & \mathscr{T}_{c}(D F)=\delta\left(\mathscr{T}_{c}(F)\right) \\
\text { ii) } & \left|\mathscr{G}_{c}^{+}(D F)-\mathscr{G}_{c}^{+}(F)\right|=\epsilon\left(\mathscr{T}_{c}(F)\right)
\end{array}
$$

In (Dii), it is the absolute difference that matters, otherwise we will get incorrect results in negative contexts.

So e.g. with $\epsilon(x)=5 x$, and a tolerance level of $2 \mathrm{~cm}$ for 'tall', we would say that John is definitely tall just in case anyone who is at most $10 \mathrm{~cm}(5 \cdot 2 \mathrm{~cm})$ shorter than John is tall as well. Also, with $\delta(x)=x / 2$, John is definitely definitely tall just in case anyone who is at most $5 \mathrm{~cm}(5 \cdot(2 / 2) \mathrm{cm})$ shorter than John is definitely tall, and hence just in case anyone who is at most $15 \mathrm{~cm}$ shorter than John is tall.

These examples agree with two intuitions about the semantic effect of 'definitely' on vague predicates that were stated above in (MoreSafe) and (LessTolerant). First, in general, someone is definitely $F$ just in case he is at a safer distance from being not- $F$ than someone who is $F$ but not definitely $F$. Minimally, at least as safe. Secondly, in general the tolerance level of the predicate $D F$ is lower than that of $F$ : the distinction between being definitely $F$ and not being definitely $F$ is finer than that between being $F$ and not being $F$. Minimally, at least as sensitive 18

Intuitions such as these provide constraints on the choice of values for $\delta$ and $\epsilon$. As for $\epsilon$, the minimally acceptable level of added safety is that of preserved safety, which means that anyone who is definitely $F$ is $F$ as well. That is, we want the T axiom

$$
D F t \rightarrow F t
$$

to be valid. In propositional modal logic, this corresponds to reflexiveness in the accessibility relation between worlds. In the scale-based approach, this corresponds to non-negative added safety.

Also, we don't want the safety margin to increase with iterations of $D$; that is, we don't want the margin between being $D D F$ and being $D F$ to be greater than the margin between being $D F$ and being $F$. Since the tolerance for a predicate $F$ is the same as for $\neg F$, and since the added safety for $D F$ is the same as the added safety (against being $F$ ) for

\footnotetext{
${ }^{18}$ The first intuition, I think, is widely shared, and almost incontrovertible. The second relates to 'definitely' in natural language, but to my knowledge has not previously been defended in the vagueness literature.
} 
$D \neg F$, the result would be as follows: allowing an increase in added safety would allow an object to be $D F$ but not $D \neg D \neg F$. For instance, suppose we have a central gap for being tall between $180 \mathrm{~cm}$ and $175 \mathrm{~cm}$. Further, we suppose that the gap for 'definitely tall' is $1 \mathrm{~cm}$, that the upper edge of the gap for being definitely tall is at $185 \mathrm{~cm}$, and for being definitely definitely tall at $205 \mathrm{~cm}$. Then you are definitely not tall if you are $170 \mathrm{~cm}$ or shorter $(175 \mathrm{~cm}-5 \mathrm{~cm})$. With the $1 \mathrm{~cm}$ gap for 'definitely not tall', anyone at $171 \mathrm{~cm}$ or taller is not definitely not tall. And then, with the added $20 \mathrm{~cm}(205 \mathrm{~cm}-185 \mathrm{~cm})$, the upper edge of the gap for definitely not definitely not tall is at $191 \mathrm{~cm}$. John, who is 186 $\mathrm{cm}$ tall is then definitely tall, but does still not qualify for being definitely not definitely not tall. This is counterintuitive: if one is definitely on the positive side, then one cannot intuitively fail to be definitely not definitely on the negative side 19

On the scale-based approach, the simplest way to block this situation is to impose a monotonicity requirement: when the tolerance level is smaller, the added safety should not increase. Together, these requirements amount to:

$$
\begin{array}{ll}
\text { i) } & \epsilon(x) \geq 0 \\
\text { ii) } \quad x \leq y \rightarrow \epsilon(x) \leq \epsilon(y)
\end{array}
$$

Turning to the $\delta$ function, we take 'definitely' to modify tolerance, but neither to introduce tolerance nor to eliminate tolerance. That is, we would take it as an empirically motivated constraint that

$$
\begin{aligned}
& \text { i) } \quad \delta(x)=0 \quad \text { if } \quad x=0 \\
& \text { ii) } 0<\delta(x) \leq x \text { if } 0<x
\end{aligned}
$$

Since all tolerance values are non-negative, the case of $i<0$ will not arise. By $[\delta]$, any predicate that is precise is also definitely precise, and so infinitely higher-order precise: if the tolerance of $F$ is zero, the tolerance of $D F$ is zero as well. Any predicate that is vague, i.e. has non-zero tolerance, is infinitely higher-order vague: for if the tolerance of $F$ is non-zero, then the tolerance of $D F$, is non-zero, and hence $D^{n} F$ for any $n$ has non-zero tolerance. We shall return to these issues below.

The second clause requires that tolerance for 'definitely $F$ ' is not higher than the tolerance of $F$. Minimally, 'definitely' then preserves the tolerance of what it applies to. The tolerance principle for $D F$ requires that objects having an $\mathscr{H}_{c}(F)$ measure at least $\delta\left(\mathscr{T}_{c}(F)\right)$ below any object that is $D F$ is also $D F$. Should then $\epsilon$ be the the constant zero

\footnotetext{
${ }^{19}$ In fact, we can allow the safety margin to increase without violating $\mathrm{T}$, provided the safety margin for $D F$ is not greater than the sum of the safety margin and the tolerance for $F$. If this were to be violated, the $\mathrm{B}$ axiom schema will not be valid (see below).
} 
function, i.e. $\epsilon\left(\mathscr{T}_{c}(F)\right)=0$, then if $\delta(y)>y$, the gaps for predicates with iterated applications of $D$ will keep growing, in the end possibly forcing a domain cut that leaves nothing in the domain. The simplest and most natural way of avoiding this is to impose a nongrowth requirement.

How does this work in discrete orders? In discrete orders, there is a smallest difference, such as a difference of one hair as between two bald men? Say that Bill has no hairs. Then Bill is bald, definitely bald, and so on. He is definitely ${ }^{n}$ bald for any positive $n$. Suppose for simplicity that nobody in the domain has exactly one hair, while Tom has two. Tom is definitely ${ }^{k}$ bald, for some positive $k$, but not definitely ${ }^{k+1}$ bald. Now it does hold that for any $j>k$, if A has $x+1$ hairs and is definitely ${ }^{j}$ bald, and B has $x$ hairs, then $\mathrm{B}$ is definitely ${ }^{j}$ bald as well. So tolerance is preserved for iterations of 'definitely'. Above a certain number of iterations, the tolerance level does not shrink any more, but does not reduce to zero either. This works because we are allowed zero added safety: for $j>k$, anyone who is definitely ${ }^{j+1}$ bald is not more safe from being non-bald than anyone who is definitely ${ }^{j}$ bald. With smaller number of iterations, we will have both positive added safety and shrinking tolerance ${ }^{20}$

The structure of the extensions and anti-extensions of predicates formed by means of $D$ and $\neg$ is illustrated in Figure 2. The illustration accords with the restrictions on $\epsilon$ and $\delta$. Only the vertical dimension matters; the horizontal spread is only for legibility. The filled rectangles represent the gaps of $F, D F, D \neg F$, and $D \neg D \neg F$, respectively.

This completes the presentation of the basic ideas. Before going on with the formal semantics, I'll briefly discuss the conception of definiteness.

\section{On the interpretation of 'definitely'}

Usually, with respect to a gradable adjective like 'tall', where an object is placed on a scale matters to whether it qualifies for definitely satisfying the adjective (or the corresponding predicate). Someone who is definitely tall is typically taller than someone who is just tall but not definitely tall (all with respect to the same context, comparison class, etc.). Still, the literature on higher-order vagueness tends to treat 'definitely' (and 'clearly') not as characterizing a position on a scale, but as characterizing a way for a sentence or proposition to be true. This is reflected in the axiom schemata for the the operator, especially (PC) and $\underline{\mathrm{RN}}$ :

(PC) If $\phi$ is a truth-functional tautology, then $=\phi$

\footnotetext{
${ }^{20}$ It should be noted, though, that for a predicate like 'bald', with an associated discrete order, it is not the case that for any finite set of objects there is a higher-order gap model. That is, Fact 1 does not in general hold for the discrete case.
} 


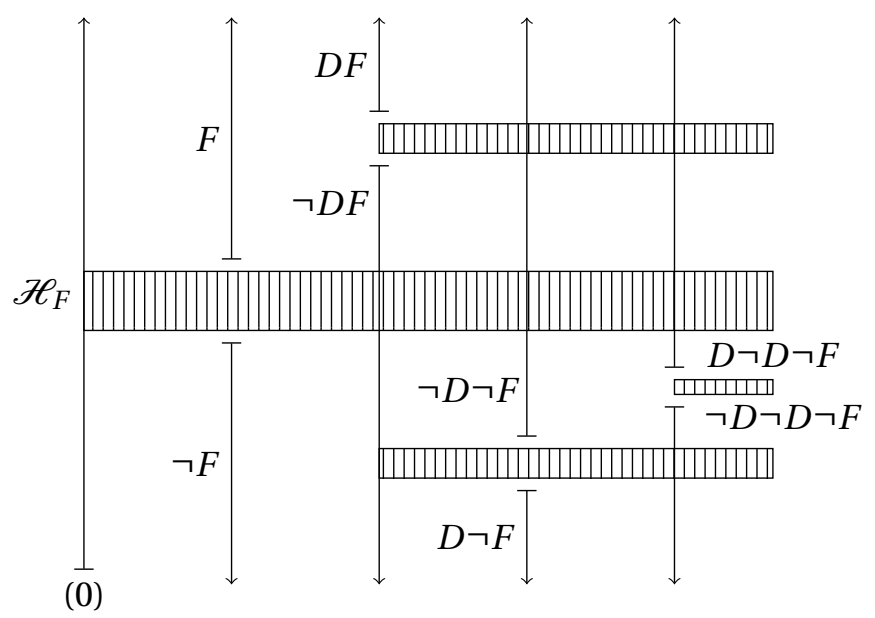

Figure 2: Definitely and not definitely.

RN If $\models \phi$, then $\models D \phi$

It may look as if the conception of 'definitely' as a predicate modifier does not fit the ideas of definiteness that are expressed in these schemata. But appearances are misleading. A tautologous predicate, such as ' $F \rightarrow F$ ' (or ' $\lambda x(F x \rightarrow F x$ )'), is logically true of every object. On the current interpretation of predicates as true "at" objects, this accounts for (PC). Also, if $F$ is true of every object, then $D F$ is true of every object, for $D F$ is true of an object $a$ just in case $F$ is true of every object within the safety margin of $a$, which holds trivially, since $F$ is true of every object anyway. The basic logical properties survive in the scale-based predicate modifier conception ${ }^{21}$

Since definiteness is usually associated with not being borderline, the idea of an object being $F$ but not definitely $F$ often has a rather peculiar status, since being borderline $F$ is sometimes seen as being incompatible with being $F$. Within supervaluationism, they are indeed inconsistent: For a sentence to be borderline in a valuation is for that

\footnotetext{
${ }^{21}$ Note that $\mathrm{RN}$ means that if $F$ is true of all objects in a domain, so is $D F$. So-called $D$-introduction, accepted by Fine (1975 p. 288), and used by Fara (2003) to generate her paradox of higher-order vagueness, is not valid. $D$-introduction allows us to infer that $D F$ holds of an object if $F$ holds for that object. This is clearly inconsistent with the idea of added (over and above preserved) safety.

Cobreros 2011a) provides a variant of Fara's paradox that does not employ D-introduction, but instead relies on arbitrarily iterated definitizations of Fara's premises. Where Fara has premise $\phi$, Cobreros has $D^{n} \phi$, for $n$ as large as is needed (for generating the paradox). The resulting set of premises is indeed unsatisfiable, also by my standards. Any of the premises can be true in a gap model, and none is true in all models. It will depend on the distribution of objects and measures in the particular model. Cobreros argues 2011a p. 220) that his opponent (any theorist who characterize vagueness in terms borderline cases - which does not include me) is committed to accepting the definitized premises. Maybe that is true, but it is certainly not true of the theorist who defends an intensifier interpretation of 'definitely'.
} 
sentence to be neither true nor false. Within epistemicism, which sticks to bivalence, there are indeed sentences that are true but not definitely true, but no sentence can be known to have this status 22

In Fine's introductory discussion (2008 pp. 113-4), he considers the unacceptability of asserting " $P$ and not definitely $P$ ". He argues that if asserting $P$ commits the speaker to $P$ being definitely the case, then asserting " $P$ and not definitely $P$ ", the speaker is committed both to $P$ being definitely the case and it's not being definitely the case. So, by reductio, the speaker who says that $P$ is not definitely the case commits himself to the claim that $P$ is not the case. This is a conclusion we want to avoid, says Fine, since if it were to hold, it would be inconsistent to claim that $P$ is borderline. His solution is that in asserting that $P$ one commits oneself to much more than the content:

In asserting some propositions $\mathrm{P}_{1}, \mathrm{P}_{2}, \ldots$, one is committed to more than their actual content, one is also committed to their being definitely the case, definitely definitely the case, definitely definitely definitely the case, and so on. We might say that a proposition is super-definitely the case if it is the case, definitely the case, definitely definitely the case, and so on ad infinitum. Then in asserting some propositions, one is also committed to their being super-definitely the case and it is because of this further content that one cannot infer from the inconsistency of $Q$ with some other propositions that Q alone (apart from its further content) is not the case. In the case of the assertion of not definitely $\mathrm{P}$ and $\mathrm{P}$, for example, what explains their joint incompatibility is the straightforward conflict between P's being definitely the case and its not being definitely the case; and no inference from not definitely $\mathrm{P}$ to not-P is therefore justified (Fine 2008 p. 114).

As regards accounting for the apparent unacceptability of asserting " $P$ and not definitely $P$ ”, Fine's idea of commitment to super-definiteness is clearly overkill ${ }^{23}$ It is enough that the speaker commits herself to the truth of "Definitely $P$ " in asserting " $P$ ", to "Definitely definitely $P$ " in asserting "Definitely $P$ ", and so on. All that would follow from asserting that $P$ is not definitely the case is that the speaker is not in a position to assert that $P$, which falls short of committing himself to $P$ 's not being the case.

This also is consonant with Williamson's view of knowledge and assertion. According to his knowledge account of assertion (cf. Williamson 2000), a speaker should assert that

\footnotetext{
${ }^{22}$ A referee draws attention to the fact, pointed out by Williamson 1994 pp. 152,297), that $\phi \wedge \neg D \phi$ is globally invalid in supervaluationism. An inference from $\Gamma$ to $\phi$ is globally valid iff $\phi$ is true on all precisifications in case all elements in $\Gamma$ are true in all precisifications. On a simple supervaluationist interpretation of $D$, $D \phi$ is true in a valuation just in case $\phi$ is true in all precisifications. Under these circumstances, $\phi \wedge \neg D \phi$ cannot be true in all precisifications, and is therefore globally inconsistent. Cf. Fine 1975 p. 283, Cobreros $2011 b$

${ }^{23}$ It is used in the proof of his Theorem 1. A referee suggests that Fine's acceptance of commitment to super-definiteness reflects his own acceptance of global validity. Although Fine accepted global validity in 1975. it is hard to see traces of such acceptance in 2008 in which supervaluationism is rejected.
} 
$P$ only if she knows that $P$, in which case $P$ is definitely true (since one cannot, according to epistemicism, know the truth of borderline sentences).

Asserting " $P$ and it is borderline whether $P$ " indeed is intuitively unacceptable, for in asserting that it is borderline whether $P$ you let it be understood that you cannot tell whether $P$, while in asserting that $P$ you let it be understood that you can tell whether $P 24$

However, as far as natural language intuitions are concerned, it is not so clear that 'not definitely and not definitely not' is equivalent with 'borderline'. Rather, it seems that 'definitely', or 'clearly', is associated with the existence of a safe margin for the correctness of an assertion. But that you can be more reliable in asserting that $P$ when it definitely true that $P$ does not rule out that you can be sufficiently reliable even if $P$ is just true, not definitely true. The natural language intuitions do not, I think (or at least not clearly), speak in favour of making 'definitely' the opposite of 'borderline'.

Nonetheless, there is still something odd about asserting

John is tall but not definitely tall

This can be naturally explained by the fact that either as a sentence operator a or predicate modifier $(\mathrm{b})$, the following cannot be true:

a. Definitely (John is tall but it is not the case that definitely (John is tall)).

b. John is definitely (tall but not definitely tall).

That these sentences cannot be true follows from the natural premise that 'definitely' distributes over conjunction, together with the factivity of 'definitely' (corresponding to the (T) axiom schema). If John is definitely (tall and not definitely tall), then, by distribution, he is definitely tall and he is definitely not definitely tall, and so, by factivity, he is not definitely tall. Contradiction.

In a sense, then, you cannot assert [11] with a safe margin. Asserting (11] is not selfdefeating, neither on the present account, nor intuitively, I think, but since it cannot be done with a safe margin, such an assertion will have a somewhat precarious status.

Before turning to the formal semantic implementation, it might be worth mentioning that the full semantics set out below for arbitrarily many iterations of $D$ is not supposed to be somehow determined by speakers' intentions in a context. We only need the semantics to cover as many iterations as are actually used (almost never more than

\footnotetext{
${ }^{24}$ Indeed, on my own account of assertion (Pagin 2011a), an assertion is an utterance that is prima facie informative, i.e. prima facie made because it is true. If the utterance $i s$ informative, then the truth of what is asserted may be correctly inferred from the assertion's having been made. So, prima facie, if you assert that $P$, you can tell whether $P$.
} 
one). To show that we can define a semantics for any finite number of iterations is meant rather as a "proof of concept", that is, as showing that the idea is sound.

\section{Semantics for the simple language}

For the gap semantics of a simple predicate $F$ we need a central $F$-gap. Since $D F$ is vague if $F$ is vague, if the language contains $D$, we will need a central $D F$-gap, as well as a central $D \neg F$-gap. At the next level, we will need 4 central gaps, for $D D F, D D \neg F, D \neg D F$, $D \neg D \neg F$. And so on. These gaps are hierarchically ordered. Figure 2 provides a view from above. In order to implement the idea, we shall associate edges of gaps with finite sequences of + and - . Any finite set of such sequences, together with empty sequence as the apex, forms a finite binary tree ${ }^{25}$ The entire infinite set corresponds to an infinite binary tree.

A + corresponds to the upper edge of a gap, and a - to a lower edge. The sequence $\langle+\rangle$ corresponds to the upper edge of the main central gap, and $\langle-,+,+\rangle$ corresponds to the upper edge of the smallest gap in Figure 2, which is the lower boundary of $D \neg D \neg F$. We define the set inductively.

Definition 1. Let $T$ be the smallest set of finite sequences such that

i) \langle\rangle$\in T$,

ii) If $\xi \in T$, then $\xi^{\curlyvee}\langle+\rangle \in T$ and $\xi^{\prec}\langle-\rangle \in T$

Here \langle\rangle is the empty sequence, and $\xi^{\prec}\langle *\rangle$ is the sequence that extends $\xi$ with $\langle *\rangle$. $\xi$ is its immediate subsequence.

In providing the semantics we shall assign real numbers, relative to scales, to the gap edges, as identified by the sequences that lead to them. We shall call these functions $T$-assignments. A T-assignment $\tau$ is a function from the set $T$ to real numbers. Such a function is relative to a tolerance function $\delta$ and safety function $\epsilon$, and so is properly written $\tau_{\delta, \epsilon}$. To avoid clutter, I shall drop the subscripts.

In the definition below, conditions i) and ii) are the conditions we have already set out for the $\delta$ and $\epsilon$ functions. $l(\xi)$ is the length of the sequence $\xi . *$ is + or - , uniformly within each sentence, and where $*$ is + , let $\overline{\text { be }}-$, and vice versa. For a any function $f, f^{1}=f$ and $f^{i+1}(x)=f\left(f^{i}(x)\right)$. The idea is that when we move from an upper edge to the next level upper edge, or from a lower edge to the next level lower edge, it is only the value of $\epsilon$ that matters. When we move from an upper edge to the next level lower edge, we jump first to the next upper edge, and then back from there to the lower edge of the

\footnotetext{
${ }^{25} \mathrm{Here}$, if $\xi$ is a sub-sequence of $\xi^{\prime}$, and both are in a set $\mathrm{V}$, the tree corresponding to $\mathrm{V}$ does not contain $\xi$ as a separate branch that ends with a leaf, but is only an initial segment of such a branch.
} 
same gap. Analogously when we move from a lower edge to the next level upper edge. In these cases, we need both the $\epsilon$ value at the given level and the $\delta$ value (the gap size) at the next level. This explains the difference between iiib) and iiic) below.

Definition 2. A function $\tau: T \longrightarrow \mathbb{R}$, relative to functions $\delta: \mathbb{R} \longrightarrow \mathbb{R}$ and $\epsilon: \mathbb{R} \longrightarrow \mathbb{R}$, is a T-assignment iff

i) a) $\delta(0)=0$

b) If $0<x$, then $0<\delta(x) \leq x$

ii) a) $\epsilon(x) \geq 0$

b) If $x \leq y$, then $\epsilon(x) \leq \epsilon(y)$

iii) a) For any $\xi \in T, \tau\left(\xi^{\frown}\langle *,+\rangle\right)-\tau\left(\xi^{\frown}\langle *,-\rangle\right)=\delta\left(\mid \tau\left(\xi^{\urcorner} *\right)-\tau\left(\xi^{\frown} \bar{*}\right)\right)$, and, where $d=\tau\left(\xi^{\wedge}\langle+\rangle\right)-\tau\left(\xi^{\wedge}\langle-\rangle\right)$,

b) For any $\xi \in T, \tau(\xi \prec\langle *, *\rangle)=\tau(\xi \prec\langle *\rangle) * \epsilon(d)$

c) For any $\xi \in T, \tau\left(\xi^{\curlyvee}\langle *, \bar{*})=\tau\left(\xi^{\curlyvee}\langle *\rangle\right) * \epsilon(d) \bar{*} \delta(d)\right.$

Here b) and c) have two variants, depending whether * is + or -. Note that the last occurrence of '*' or ' $₹$ ' in b) and c) has its mathematical meaning of addition or subtraction.

To give an example, the upper and lower edges of the third-level gap (corresponding to two iterations of $D$ in Figure 2) correspond to the sequences $\langle-,+,+\rangle$ and $\langle-,+,-\rangle$ respectively. You get the $\tau$ value for its upper edge by $\tau(\langle-,+,+\rangle)=\tau(\langle-,+\rangle)+\epsilon(\delta(\delta(z)))$, and for its lower edge by deducting $\delta(\delta(\delta(z)))$ from that value. Again, $z$ is the size of the main gap 26

Next, we turn to the syntax semantic clauses for the Simple Language, SL. In SL, we can form complex predicates by means only of the definitely and negation operators ${ }^{27}$

Definition 3. The simple language SL is a structure $\left(T_{S}, A_{S}, P_{S}, S_{S}\right)$ where $T_{S}$ is the set of terms of SL, $A_{S}$ the set of atomic (one-place) predicates of SL, $P_{S}$ the set of predicates of $\mathrm{SL}$, and $S_{S}$ the set of sentences of SL. $P_{S}$ is the smallest set that includes $A_{S}$ and such that if $F \in P_{S}$, then $\neg F \in P_{S}$ and $D F \in P_{S} . S_{S}$ is the smallest set such that if $t \in T_{S}$ and $F \in P_{S}$, then $F t \in S_{S}$.

\footnotetext{
${ }^{26}$ We shall continue to assume for simplicity that basic scales for simple predicates are upwards directed in the sense that if an object $a$ is in the extension of a predicate $F$, and object $b$ has a higher value on the relevant scale, then $b$ is in the extension as well. Should we allow the direction to vary, we would include as a third element in the central gap a direction function $\mathscr{G}^{d}$, where the values are in $\{\downarrow, \uparrow\}$.

${ }^{27}$ What I call "Complex Language" $(C L)$, adds conjunction, including conjunction under D. Giving the semantics for $C L$ must be deferred to another occasion.
} 
We shall provide a semantics for SL. A model for tolerant predicates (called "tolerance models" in Pagin 2010) requires the existence of a central gap for each predicate. When we can form complex predicates by means of $D$ and $\neg$, we need a structure of gaps, which will be provided by the $T$-assignment. For this purpose, we shall need a few new definitions.

Every predicate $F \in P^{S}$ of SL is built up from an atomic predicate $F^{\circ} \in A_{S}$, by means of $D$ and $\neg$. For an arbitrary predicate $F \in P_{S}$, let $F^{\circ}$ be the atomic predicate $F$ is based on. That is:

Definition 4. The atomic base predicate $F^{\circ} \in A_{S}$ of a predicate $F \in P_{S}$ is defined as

i) $F^{\circ}=F$, for $F \in A_{S}$

ii) $(\neg F)^{\circ}=F^{\circ}$

iii) $(D F)^{\circ}=F^{\circ}$

Then we can define what a higher-order gap model is:

Definition 5. A higher-order gap model $M$ for SL is a structure $\left(E, \llbracket \cdot \rrbracket, \delta_{F}, \epsilon_{F}, \mathscr{G}_{F}, \mathscr{H}_{F}\right)_{F \in A_{S}}$, where $E$ is a domain of individuals, $\llbracket \rrbracket$ the interpretation function, and for $F \in A_{S}, \delta_{F}$ and $\epsilon_{F}$ are functions on real numbers, $\left(\mathscr{G}_{F}\right)$ is $T$-assignment relative to $\delta_{F}$ and $\epsilon_{F}$, and $\mathscr{H}_{F}: E \longrightarrow \mathbb{R}$ a measure function on objects in $E$. It is required that

$\left(\mathscr{H}\right.$-req) $\quad$ For any $F \in P_{S}$, any $\xi \in T$, and any $a \in E, \mathscr{H}_{F^{\circ}}(a)>\mathscr{G}_{F^{\circ}}(\xi \frown\langle+\rangle)$ or $\mathscr{H}_{F^{\circ}}(a)<$ $\left.\mathscr{G}_{F^{\circ}}(\xi\urcorner\langle-\rangle\right)$.

According to $\mathscr{\mathscr { C } \text { -req }}$, for each measure function $\mathscr{H}_{F}$ in a higher-order gap model $M$, and any object $a$ in $E_{M}$, the value of $\mathscr{H}_{F}$ for $a$ is not in any gap in the hierarchy of gaps in the model. This is what ensures that we can consistently affirm tolerance principles for every predicate in SL.

Do higher-order gap models exist? Yes. We can show that for any atomic predicate $F$, any finite set of objects as $E$, and any measure function $\mathscr{H}_{F}$, there is a higher-order gap model where $(\mathscr{H}$-req $)$ is satisfied. The proof of the following Fact is in Appendix A

Fact 1. For any finite domain $E$, any $F \in A_{S}$, and any real-valued measure function $\mathscr{H}_{F}$, there are $\mathscr{G}_{F}, \delta_{F}$ and $\epsilon_{F}$ such that $\mathscr{H}$-req is satisfied.

The main idea of the proof is just to give a procedure for constructing a gap function that satisfies $\mathscr{H}$-req, given a finite set of objects and a set of measure functions for the predicates.

In order to set out the semantics, we will need to relate predicates of various complexity to the tolerance and safety levels. For this purpose, we define 
Definition 6. Each higher-order gap model $M$ is characterized by a tolerance function $\mathscr{T}$ and safety function $\mathscr{S}: P_{S} \longrightarrow \mathbb{R}$, recursively defined from $\left(\delta_{F}, \epsilon_{F}\right)_{F \in A_{S}}$ :

$(\mathscr{T}) \quad$ i) $\quad \mathscr{T}(F)=\mathscr{G}_{F}(\langle+\rangle)-\mathscr{G}_{F}(\langle-\rangle)$, for atomic $F$

ii) $\mathscr{T}(\neg F)=\mathscr{T}(F)$

iii) $\mathscr{T}(D F)=\delta_{F^{\circ}}(\mathscr{T}(F))$

$(\mathscr{S}) \quad$ a. $\quad \mathscr{S}(F)=\left|\mathscr{G}_{F}(\langle+\rangle)-\mathscr{G}_{F}(\langle-\rangle)\right|$, for atomic $F$

b. $\mathscr{S}(\neg F)=\mathscr{S}(F)$

c. $\quad \mathscr{S}(D F)=\epsilon_{F^{\circ}}(\mathscr{T}(F))$

Each gap model represents a possible context of use, so there is no further representation of context dependence in the model theory. We can now give the Simple Language Semantics. Subscripts for model, except for the first clause, will also be dropped.

Definition 7. The Simple Language Semantics SLS is given by:

i) $\quad=_{M} F t$ iff $\llbracket t \rrbracket_{M} \in \llbracket F \rrbracket_{M}$

ii) $\llbracket F \rrbracket=\left\{a \in E: \mathscr{H}_{F}(a)>\mathscr{G}_{F}(\langle+\rangle)\right\}$, for atomic $F$

iii) $\llbracket \neg F \rrbracket=E-\llbracket F \rrbracket$

iv) $\llbracket D F \rrbracket=\left\{a \in E: \forall b \in E\left(\left|\mathscr{H}_{F}(a)-\mathscr{H}_{F}(b)\right| \leq \mathscr{S}(D F)\right) \longrightarrow b \in \llbracket F \rrbracket\right\}$

Clause (iv) gives $D$ a necessity-like interpretation, as e.g. in Fine and Williamson. As noted above, a main difference is that the accessibility relation between objects depends on an aspect of the meaning of the predicate, i.e. the argument to $D$. As we shall see, this has some consequences for the logic.

We can now show that the semantics indeed does validate tolerance. The proof of the following Fact is in Appendix B.

Fact 2. For any higher-order gap model $M$ for SL and any predicate $F \in P_{S}$, for any $a \in E$, if $a \in \llbracket F \rrbracket$, then for any $b \in E$, if $\left|\mathscr{H}_{F}(a)-\mathscr{H}_{F}(b)\right| \leq \mathscr{T}(F)$, then $b \in \llbracket F \rrbracket$.

This completes the presentation of the semantics. In case we only consider predicates within the current fragment, we can also add a sentential definitely operator $\Delta$ and sentential negation $\sim$, such that

$$
\begin{array}{lll}
\text { i) } & \models_{M} \Delta(F t) \text { iff } \models_{M}(D F t) \\
\text { ii) } & \models_{M} \sim(F t) \text { iff } \quad \models_{M} \neg F t
\end{array}
$$

In this restricted case, the results easily carry over to the sentential level, but this will not work in general, for there is no obvious way to interpret e.g. ' $\Delta \sim(F t \& G u)$ '. 


\section{Simple tolerance logic}

Since we allow operators on predicates we can investigate logical properties of predicates. That is, we can define a consequence relation $1=$ for predicates, in analogy with the relation between sentences. We can define:

Definition 8. The consequence relation for predicates in (SLS) is given by

$(\mathrm{STL}) \quad$ i) For a gap model $M, \models_{M} F \quad$ iff $\quad \llbracket F \rrbracket_{M}=E_{M}$

ii) $\quad \Gamma=_{M} F$ iff $\bigcap\left\{\llbracket G \rrbracket_{M}: G \in \Gamma\right\} \subseteq \llbracket F \rrbracket_{M}$

iii) $\Gamma \models F \quad$ iff $\left.\quad \Gamma\right|_{M} F$, for all higher-order gap models $M$.

The second clause says that $F$ is consequence of $\Gamma$ in a gap model $M$ just in case the intersection of the extensions of all elements of $\Gamma$ is a subset of the extension of $F$. Hence, if any object is in the extension of all predicates in $\Gamma$, it is also in the extension of $F$.

Given the (SLS) semantics and the (STL) definition of logical concepts, we can check the validity of modal logic rules and axioms reinterpreted as a logic for predicates. However, SL does not contain any connectives (binary logical propositional constants), and so sentences that contain $\rightarrow$ do not have predicate counterparts in SL. We can to some extent circumvent this problem by restating axioms with implications in the meta-language. This makes sense, since the deduction theorem holds in modal logic 28 Therefore, for instance, the T axiom, $\vDash=D \phi \rightarrow \phi$, holds iff it also holds that that $D \phi \models \phi$. We shall then regard $D F=F$ as the tolerance logic counterpart to $\mathrm{T}$, and we can apply the same idea to the other axioms and rules that contain $\rightarrow$, unless they contain $\rightarrow$ in the scope of $D$, as does K. For more on $\mathrm{K}$, see below. What we shall do, therefore, is to check whether (SLS) satisfies the predicate counterparts. Note that for SL, the simple language, PC is inapplicable, since no predicate tautology can be expressed in SL.

The proof of the following Facts are in Appendix C.

Fact 3. SLS) validates a) $\mathrm{T}, \mathrm{b}$ ) $\mathrm{RN}, \mathrm{c}$ ) $\mathrm{B}$, and d) modus ponens.

As noted above (note21, so-called D-introduction,

$$
\vDash_{M} F x \Rightarrow \vDash_{M} D F x,
$$

is not valid, because prefixing $D$ may induce non-zero added safety. More interestingly,

$$
F\left|=_{M} G \Rightarrow D F\right|{ }_{M} D G
$$

\footnotetext{
${ }^{28}$ See Negri 2011 p. 525. We need the restriction that the rule of necessitation is valid only from derivations without assumptions. For some systems of modal logic, weaker restrictions apply.
} 
is not valid either, this time because with iterations of $D$, the added safety may shrink. Thus, if there are fewer iterations of $D$ in $G$ than in $F$, adding one to each predicate may have a smaller effect on the safety added from $F$ to $D F$ than on the safety added from $G$ to $D G$.

On the other hand, if $\left.F\right|_{M} G$ is true in every model $M$, then $\left.D F\right|_{M} D G$ is true in every model as well. This depends chiefly on the monotonicity of the $\epsilon$ and $\delta$ functions. The proof of the following facts are in Appendix $D$.

\section{Fact 4.}

a) $\left.\left.\quad F\right|_{M} G \Rightarrow D F\right|_{M} D G$

b) $F=G \quad \Rightarrow \quad D F \models D G$

In the simple language SL, schema K cannot be expressed. Since implication occurs in the scope of $D$, K does not have a simple tolerance logic counterpart. However, let us provisionally add the complex predicate $F \rightarrow G$ by means of

(Cond) $\quad \llbracket F \rightarrow G \rrbracket_{M}=\left(E_{M}-\llbracket F \rrbracket_{M}\right) \cup \llbracket G \rrbracket_{M}$

That is, an object in $E$ is in $\llbracket F \rightarrow G \rrbracket$ iff it is in $\llbracket G \rrbracket$ or not in $\llbracket F \rrbracket$. Then, as a consequence (Include) $\quad \models_{M} F \rightarrow G \quad$ iff $\quad F=_{M} G \quad$ iff $\quad \llbracket F \rightarrow G \rrbracket_{M}=E_{M}$

Hence, by RN, we have

(Def-Incl) $\quad F \mid=_{M} G \quad$ iff $\quad \models_{M} D(F \rightarrow G)$

Now putting Fact 4 . (Include), and (Cond) together, it is clear that there is a model $M$ such that

$$
\vDash_{M} D(F \rightarrow G) \quad \text { but } \quad \nvdash_{M} D F \rightarrow D G
$$

Therefore,

Fact 5. SLS does not validate K.

Hence, the modal logic will not be normal. In models where $\epsilon$ is a constant function, K still holds 29

\footnotetext{
${ }^{29}$ The failure of $\mathrm{K}$, depending on the decreasing added safety of iterations of $D$, is sufficient for blocking the impossibility results in Fine 2008
} 


\section{Infinite higher-order vagueness}

For Williamson (1999), a consequence of accepting the logic B (KTB), together with his formal definition of a vague formula, is that second-order vagueness entails higher-order vagueness of all finite orders (1999 p. 136), which is also a central claim of that paper. Translated into the present terminology, this means that if an object $a$ is e.g. neither in $\llbracket D D F \rrbracket$ nor in $\llbracket D \neg D F \rrbracket$, then for any number $k \geq 2$, there is a predicate $G$ with $k$ occurrences of $D$, such that $a$ is in neither $\llbracket D G \rrbracket$ nor in $\llbracket D \neg G \rrbracket$.

In order to facilitate the comparison with the present framework, I'll give a sketch of an alternative proof of Williamson's result. In Williamson's framework, second-order vagueness presupposes first-order vagueness. Let $w$ be a $\phi$ world that is borderline $\phi$. Then there is a $w$-accessible world $w^{-}$where $\neg \phi$ is true. Also, let $w$ be a borderline for $D \phi$ : Then, $\neg D D \phi \& \neg D \neg D \phi$ is true at $w$. Then there is a $w$-accessible world $w^{+}$where $D \phi$ is true.

Now, $D \neg D \phi$ is true at $w^{-}$. This is so because accessibility is symmetric. If there were a world $w^{\prime}$ accessible from $w^{-}$where $D \phi$ is true, $\phi$ would be true in $w^{-}$. Hence, $\neg D \phi$ is true in all worlds accessible from $w^{-}$, and therefore $D \neg D \phi$ is true at $w^{-}$. Also, since $D \phi$ is true at $w^{+}, \neg \neg D \phi$ is true at $w^{+}$.

Now, set $\psi=\neg D \phi$. We then have the situation that $\neg \psi$ true at $w^{+}$and $D \psi$ true at $w^{-}$. Because of the latter, by reflexivity (T), $\psi$ is true at $w^{-}$, and hence $w$ is borderline $\psi$. Suppose that $D D \psi$ is true at $w$. If so, $D \psi$ is true at $w^{+}$, and again by reflexivity, $\psi$ is true at $w^{+}$, contrary to the definition of $\psi$. Hence, $\neg D D \psi$ is true at $w$. Suppose that $D \neg D \psi$ is true at $w$. If so, $\neg D \psi$ is true $w^{-}$, contrary to the definition of $\psi$. Hence, $\neg D \neg D \psi$ is true at $w$. And so, $w$ is borderline $D \psi$.

We can therefore repeat the reasoning above with $\psi$ instead of $\phi$, and the roles of $w^{+}$ and $w^{-}$reversed. And so on.

This result depends on the symmetry of the accessibility relation, on Williamson's preferred definition of higher-order vagueness, and on the fact that the accessibility relation is absolute (not relative to a sentence). Without the validity of $\mathrm{K}$, Williamson's claim is not true 30

As far as I can see, Williamson's result does not address the intuition behind higherorder vagueness. The intuition was that when we try to seek new boundaries between

\footnotetext{
${ }^{30}$ Mahtani 2008 objects to Williamson's symmetry assumption. She argues that in Williamson's framework, 'definitely' must itself be seen as vague. The interpretation of 'definitely' at a point determines how close another world must be in order that interpretations are indistinguishable. Therefore, the interpretation of a language $L$ at point $i$ might be indistinguishable from the interpretation of $L$ at point $j$, but not conversely, due to the fact that the interpretation of 'definitely' at $j$ is more strict. As far as I can see, this kind of model is perfectly acceptable, but it is also possible to allow for a variation in the strictness of 'definitely' that is still consistent with symmetry, as long as the variation is not very steep.
} 
regions not so far considered, we will in each case find the boundaries unsharp; there will always be actual or potential new borderline cases. Williamson's result trades on the fact that the very same world can keep being borderline a case because more complex formulas according to a selected pattern actually characterize the very same region 31

In the current setup, the argument breaks down at the repetition step. Unlike in Williamson's framework, we cannot infer that $w$ is borderline $\psi$. That would require that $\neg D \psi$, i.e. $\neg D \neg D \phi$, is true at $w$, but it need not be. For although $\neg \psi$ is true at $w^{+}$and $w^{+}$ is $\phi$-accessible from $w, w^{+}$need not be $\psi$-accessible from $w$. So $D \psi$ may in fact be true at $w$.

How then does higher-order vagueness develop in the present framework, beyond second-order vagueness? We have already seen that tolerance does not disappear at any finite number of iterations of $D$. So if were to define higher-order vagueness as non-zero tolerance of predicates with finitely many iterations of $D$, we will have infinite higherorder vagueness.

The matter stands differently if we use Fine's (1975) or Williamson's (1999) definitions. For suppose that we have a domain with finitely many objects, say $n$. Now, in order that $\neg D F$ be true of an object $a$, it must not be true that all objects $x$ within a distance $\epsilon(\mathscr{T}(F))$ are $F$. That requires the existence of an object $b$ within that distance that is $\neg F$. Similarly, if an object $c$ is $\neg D \neg D F$, there must be an object $d$ within a distance of $\epsilon(\delta(\mathscr{T}(F)))$ that is $D F$, and so on. In order that predicates of the form $\neg D X$ be true, objects of which $\neg X$ are true must exist. With definitions like Fine's or Williamson's, higher-order vagueness has existential import. Now, with shrinking tolerance and safety levels, new objects will be needed to populate regions defined by the conditions of being $X$ but not $D X$. Since these regions will be denumerably many, and only finitely many will be inhabited, it will hold for infinitely many predicates $X$ (with many iterations of $D$ ) that $\forall y(D X(y) \vee D \neg X(y))$ is true, not because $X$ is sharp, but because it so happens that every object that is $X$ is also $D X$ and every object that is $\neg X$ is also $D \neg X$. So because of the existential import of higher-order vagueness, on definitions of this type, in finite domains, higher-order vagueness will run out at some finite level ${ }^{32}$

\footnotetext{
${ }^{31}$ Fara 2003 p. 205) has a related observation, claiming that a set of three objects is enough to demonstrate the possibility of infinite higher-order vagueness without fuzzy boundaries. In her case, the idea is that for any $k$, ' $D$ k tall' is true of the first object, false of the third, and neither true nor false of the second.

Somewhat related, too, is Bobzien's idea 2013 p. 15) columnar higher-order vagueness, in which we only have the original three areas: $D F, D \neg F$, and $\neg D F \wedge \neg D \neg F$; any larger combination of iterations of $D$ and $\neg$ will coincide with one of these three.

Indeed, we get precisely this result in the intensifier framework in case $\delta(x)=x$ and $\epsilon(x)=0$ : tolerance doesn't shrink, and safety isn't added. From the perspective of $D$ as an intensifier, this possibility is not of central importance.

${ }^{32}$ The Higher-order sorites paradox in Zardini 2013 employs a premise called (R) 2013 p. 38, which says
} 
There is, however, a further, perhaps more interesting aspect of infinite higher-order vagueness. Even if we fix the parameters for $\delta$ and $\epsilon$ so that both tolerance and safety decreases, this does not by itself settle the question whether there is a maximum infiniteorder safety. For it is left open whether the infinite series

$$
\mathscr{S}(F)+\mathscr{S}(D F)+\mathscr{S}(D D F)+\ldots
$$

i.e.

$\left(\infty^{\prime}\right) \quad \sum_{i=0}^{\infty} \mathscr{S}\left(D^{i}(F)\right)$

converges or diverges. If it diverges, then for any possible object $a$ there is a number $k$ of iterations such that $D^{k} F$ is not true of $a$.

Suppose, on the other hand, the series does converge to some finite value $l$. Then there can be an object $a$ with $\mathscr{H}_{F}(a)>l$, such that for any number $k, D^{k}(F)$ is true of $a$. It is reasonable to define this as being infinitely definitely $F^{33}$

\section{$\left(D^{\omega}\right) \quad D^{\omega} F(a)$ iff for any $k, D^{k} F(a)$}

There are two kinds of infinite definiteness. The first kind comprises those examples where the added-safety function $\epsilon$ yields a positive value for any finite number of iterations. In examples of this kind, since the sum $l$ of a convergent series is a limit value, it holds of any real number $j<l$ that if $\mathscr{H}_{F}(b)=j$, then it is not the case that $D^{\omega} F(b)$. Because of this, $D^{\omega}$ has zero tolerance, and it is therefore a sharp predicate. That is, vagueness in the sense of tolerance may peter out along a dimension of variation.

The second kind comprises those examples where the $\epsilon$ yields zero after a finite number of iterations, and then continues giving zero as value for any additional finite number. In such examples, there will be natural number $n$ such that $D^{n} F \leftrightarrow D^{\omega} F$. The

"For every $i$, ' $D^{i} B^{\prime}$ is definitely ${ }^{\omega}$ vague". Zardini argues for this principle by appeal to epistemic limitations: there is no number $n$ such that we have the capacity to identify sharp boundaries for predicates with $n$ iterations of $D$. However, (R) is then used to justify premises like Premise 2 : “ $D^{999,999} \neg \exists x\left(D D B x \wedge D \neg D B x^{\prime}\right)$ " (where $x^{\prime}$ is the object directly following $x$ in a sorites sequence, 2013 p. 39).

From the present perspective, this move is illicit. The claim concerning epistemic limitations is correct, for at no finite number of iterations does tolerance reduce to zero. The conclusion drawn, however, Premise 2 , requires there to be objects in the domain to fill up the relevant regions. So an extra assumption about the domain of discourse is needed. This assumption is met Zardini's own case, where the domain is the set of natural numbers between 0 and 1,000,000 2013 p. 28). In higher-order Gap models, principles like Premise 2 will be false in case the successor operator ' ${ }^{\text {is }}$ understood as mapping any (non-final) object in an appropriately ordered sequence on the object that succeeds it. For with suitable gaps, the next object may in fact be not so similar in the relevant measure. If, instead we keep the usual interpretation of ${ }^{\prime}$ as the natural number successor operation, and the model has gaps, then it will not be defined for every argument.

${ }^{33}$ Fine 2008 p. 114 calls it "super-definitely". 
example of 'bald' discussed in Section 4 , would be a case in point. In examples of this kind, $D^{\omega} F$ is not sharp.

In both kinds of examples, since the series converges, it must be the case that $\epsilon\left(\mathscr{T}\left(D^{k} F\right)\right)$ approaches 0 as $k$ increases. Therefore, $\epsilon\left(\mathscr{T}\left(D^{\omega}\right)\right)=0$. Hence, if an object $a$ is $D^{\omega} F$, it is also $D D^{\omega} F, D D D^{\omega} F$, etc. In fact, it is $D^{\omega} D^{\omega} F$, and so on 34

Whether the series $\infty$ ) converges or not depends on the functions $\epsilon$ and $\delta$. For instance, if $\epsilon$ is a linear function, such as $2 k$, and $\delta$ is a decreasing exponential function such as $2^{-k}$, then $\infty$ does converge. If $\delta$ instead decreases slowly (like $1^{-k}$ ), the series will diverge.

We could require as part of the meaning of $D$ that the series $\infty$ converges. What would vary with context would then only be the limit point on the scale where tolerance disappears. Or we could leave it open, to allow the series to converge in some contexts but not in others. I don't find any of these alternative more intuitive than the others. As regards natural language, it is not to be expected that the use of 'definitely' or 'clearly' settles this question in very many contexts. We hardly ever encounter more than one iteration (i.e. one occurrence embedded under another) in natural language use.

\section{A glimpse of connectives}

So far we have restricted attention to the simple language SL, i.e. to predicates that are either atomic, or formed from atomic predicates by means of $D$ and $\neg$. The complex language, CL, allows conjunction as well. This is a considerable complication. What does it mean to say that someone is e.g. not definitely (tall and thin), or definitely not definitely (tall and thin)? Here, there is not space enough to treat this complication in any depth. I shall just indicate the very idea.

Already without the $D$ operator, we needed a two-dimensional illustration (Figure 1 on page 11] for depicting the union of gaps in contexts where more than one predicate is used. At that point, a multi-dimensional framework was not needed in the semantics, since connectives occurred only at sentence level, not at predicate level. When we add them as predicate operators, however, a multi-dimensional treatment is required.

Intuitively, for the conjunction of atomic predicates $F \wedge G$ there must be a central gap as illustrated in Figure 1 separating any object $a$ of which the predicate is true from the objects that are not $F$ and from the objects that are not $G$. As in the one-dimensional case, negation maps this are on the complement in the plane on the other side of the

\footnotetext{
${ }^{34}$ This is in agreement with Williamson 1994 p. 160 (for $D^{*}$ ) and Fine 2008 p. 122 . According to Fine, this follows by logical principles when Superdefinitely $\mathrm{P}$ is defined (like Williamson's $D^{*}$ ) as an infinite conjunction (P \& Definitely P \& ...), because definitely collects over conjunction. Note that the result here, for the tolerance operator, does not depend on using an infinitary formula.
} 


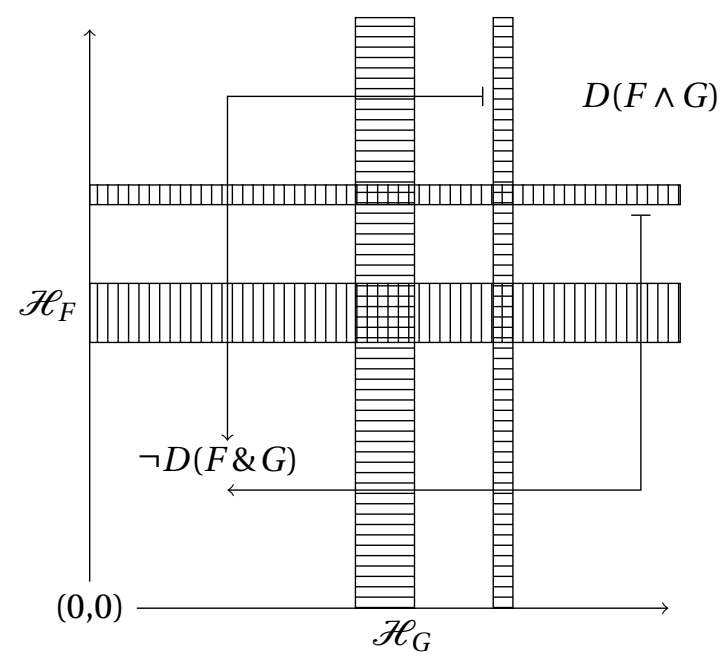

Figure 3: $D(F \wedge G)$ and $\neg D(F \wedge G)$.

gap, i.e. on the union of $\llbracket \neg F \rrbracket$ and $\llbracket \neg G \rrbracket$. Applying $D$ to such a predicate should have the effect of restricting the $F \wedge G$ area to an area that is at a safer distance both from $\llbracket \neg F \rrbracket$ and $\llbracket \neg G \rrbracket$. Figure 3 illustrates this. A full semantic treatment of this idea must await another occasion 35

\section{Conclusion}

The main aim of this paper has been suggest a tolerance-friendly account higher-order vagueness by means of a semantics for the $D$ operator as an intensifier. I have combined the account of $D$ with my previously suggested gap semantics for vague predicate. I have tried to show that the result is logically sound and accounts for arbitrarily many iterations of $D$. I have also tried to make it plausible that this account of $D$ is conceptually close to the basic idea of vagueness as a semantic phenomenon.

\section{Department of Philosophy}

\section{Stockholm University}

\footnotetext{
${ }^{35}$ Interestingly, Richard Dietz has suggested that the domain cut for $D \vee G$ should be formed by from the $F$ and $G$ cuts by removing from the union the parts that stretch beyond the intersection. This means that an object $a$ is $F \vee G$ if it is $F$ even if borderline $G$ (i.e. in the $G$ domain cut) or $G$ but borderline $F$. If an object is $\neg F$ and borderline $G$, it is in the domain cut of $F \vee G$, and so the sentence $F t \vee G t$ will be neither true nor false. The resulting semantics for compounds is Kleene's strong three-valued logic. My ambition has been to keep bivalence and classical logic within each model, not least since it makes quantification straightforward.
} 


\section{Appendices}

\section{A. Proof of Fact 1}

For any finite domain $E$, any $F \in A_{S}$, and any measure function $\mathscr{H}_{F}$, there are $\mathscr{G}_{F}, \delta_{F}$ and $\epsilon_{F}$ such that $\mathscr{H}$-req is satisfied.

Proof. Note first that if the domain is empty, $\mathscr{H}$-req is trivially satisfied. For nonempty domain, we proceed by induction on the length of $T$ sequences. The idea is to show that we can construct $\mathscr{G}_{F}, \delta_{F}$ and $\epsilon_{F}$, step by step, so as to make sure that $\mathscr{H}$-req is met. Given $\mathscr{H}_{F}$ and $E$, we have a finite set $V$ of real numbers such that $V=\left\{\mathscr{H}_{F}(a): a \in E\right\}$.

For the base case, with $l(\xi)=1$, we need to find a pair of real numbers $i, j$, with $i>j$, such that for any $r \in V, r>i$ or $r<j$. This can clearly be done: pick any elements of $V$ that are adjacent in the natural order, and pick $i$ and $j$ between these two, with $i>j$. Then set $\mathscr{G}_{F}(\langle+\rangle)=i$ and $\mathscr{G}_{F}(\langle-\rangle)=j$.

For the inductive step, assume that $\mathscr{H}$-req is satisfied for sequences of length $k$. There are $2^{k+1}$ sequences $\xi \prec\langle *\rangle$, where $l(\xi)=k$. Again, let $*$ be - if $*$ is + , and vice versa. For each such $\xi$, we need extend $\xi^{\wedge}\langle *\rangle$ into $\xi^{\wedge}\langle *,+\rangle$ and $\xi^{\wedge}\langle *,-\rangle$ such that, where $\left.d=\mid \mathscr{G}_{F}(\xi\urcorner\langle *\rangle\right)-\mathscr{G}_{F}(\xi\urcorner\langle\overline{ }(\bar{*})|$,

$$
\begin{aligned}
& \text { i) } \quad \mathscr{G}_{F}\left(\xi^{\curlyvee}\langle *,+\rangle\right)-\mathscr{G}_{F}\left(\xi^{\wedge}\langle *,-\rangle\right)=\delta(d) \\
& \text { ii) } \left.\left.\mid \mathscr{G}_{F}(\xi\urcorner\langle *, *\rangle\right)-\mathscr{G}_{F}(\xi\urcorner\langle *\rangle\right) \mid=\epsilon(d) \\
& \text { iii) }\left|\mathscr{G}_{F}\left(\xi^{\wedge}\langle *, \bar{*}\rangle\right)-\mathscr{G}_{F}\left(\xi^{\wedge}\langle *\rangle\right)\right|=\epsilon(d) \bar{*} \delta(d)
\end{aligned}
$$

in accordance with clauses iiia) - iiic) of Definition 2 . To do this, we need to find two numbers $\alpha$ and $\beta$ such that for any of the $2^{k+1}$ sequences $\xi^{\wedge}\langle *\rangle$ :

(\#\#) For no $r \in V$ does it hold that either

$$
\begin{aligned}
& \text { i) } \mathscr{G}_{F}\left(\xi^{\frown}\langle+\rangle\right)+\alpha \leq r \leq \mathscr{G}_{F}\left(\xi^{\frown}\langle+\rangle\right)+\beta \\
& \text { ii) } \left.\left.\quad \mathscr{G}_{F}(\xi\urcorner\langle-\rangle\right)-\alpha \geq r \geq \mathscr{G}_{F}(\xi\urcorner\langle-\rangle-\beta\right)
\end{aligned}
$$

Since $\beta-\alpha$ can be arbitrarily small, this can again be done: Pick $\beta$ such that for no $r \in V$, $\mathscr{G}_{F}\left(\xi^{\wedge}\langle+\rangle\right)+\beta=r$, nor $\mathscr{G}_{F}\left(\xi^{\urcorner}\langle-\rangle\right)-\beta=r . \beta$ must in addition be chosen such that

$$
\text { (†) } \quad \beta \leq \epsilon\left(d^{\prime}\right)
$$

for $d^{\prime}$ defined below, in order that clause iib) of definition 2 is satisfied (we return this below).

Then pick some $\alpha<\beta$. If some $r^{\prime} \in V$ satisfies either (i) or (ii), for some sequence $\xi$ of length $k$, then replace $\alpha$ by $\alpha^{\prime}$ such that $\alpha<\alpha^{\prime}<b$ and $\alpha^{\prime}$ does not satisfy \#\#) with 
respect to $\xi, \beta$, and $r^{\prime}$. This is possible, since $\beta-\alpha^{\prime}$ can be arbitrarily small. Repeat the procedure until you have a number $\alpha^{*}$ such that no $r \in V$ satisfies \#\# with respect to $\alpha^{*}$ and $\beta$. Since $V$ is finite and there are $2^{k+1}$ sequences to consider, this process will come to an end.

Then extend the definition of $\epsilon$ by setting $\epsilon(d)=\beta$. Preliminarily extend the definition of $\delta$ by setting $\delta(d)=\beta-\alpha^{*}$. We must check that clauses i) and ii) of Definition 2, are met. Let $\xi=\xi^{\prime \wedge}\langle+\rangle$. Let $d^{\prime}=\mid \mathscr{G}_{F}\left(\xi^{\prime}\langle+\rangle\right)-\mathscr{G}_{F}\left(\xi^{\prime}\langle\langle-\rangle) \mid\right.$. For clause ib) of 2 it must hold that $\delta(d) \leq d$. If not, choose $\alpha^{\prime}$ such that $\alpha^{*}<\alpha^{\prime}<\beta$, that meets this condition. It will also satisfy \#\#, since it is smaller than $\beta$. Let $\alpha^{\prime}$ be the final choice. That is, we finally extend the definition of $\delta$ by setting $\delta(d)=\beta-\alpha^{\prime}$.

We must also check that clause iib) is met. This holds if $\epsilon(d) \leq \epsilon\left(d^{\prime}\right)$, since by the ind. hyp. $d \leq d^{\prime}$ (because by the ind. hyp. $d=\delta\left(d^{\prime}\right)$ ). Since $\beta$ was chosen to satisfy $\dagger$, and $\epsilon(d)=\beta$, this condition is met.

Finally, extend $\mathscr{G}_{F}$ by setting

$(\# \# \#) \quad$ a) $\quad \mathscr{G}_{F}\left(\xi^{\wedge}\langle+,+\rangle\right)=\mathscr{G}_{F}\left(\xi^{\urcorner}\langle+\rangle\right)+\beta$

b) $\mathscr{G}_{F}\left(\xi^{\curlywedge}\langle+,-\rangle\right)=\mathscr{G}_{F}\left(\xi^{\curlywedge}\langle+\rangle\right)+\alpha^{\prime}$

c) $\left.\mathscr{G}_{F}(\xi \prec\langle-,+\rangle)=\mathscr{G}_{F}(\xi\urcorner\langle-\rangle\right)-\alpha^{\prime}$

d) $\mathscr{G}_{F}\left(\xi^{\curlywedge}\langle-,-\rangle\right)=\mathscr{G}_{F}\left(\xi^{\curlyvee}\langle-\rangle\right)-\beta$

We can verify that (ii)-(iii) of (\#) are satisfied. In case (iii), with $*$ as + , we have:

$$
\begin{array}{ll}
\left|\mathscr{G}_{F}\left(\xi^{\curlyvee}\langle+,-\rangle\right)-\mathscr{G}_{F}\left(\xi^{\urcorner}\langle+\rangle\right)\right| & = \\
\left|\mathscr{G}_{F}\left(\xi^{\curlywedge}\langle+\rangle\right)+\alpha^{\prime}-\mathscr{G}_{F}\left(\xi^{\urcorner}\langle+\rangle\right)\right| & = \\
\alpha^{\prime}=\beta-\delta(d) & =\epsilon(d)-\delta(d) .
\end{array}
$$

The other cases are similar.

Finally, we verify that $\mathscr{H}$-req is satisfied for $\mathscr{G}_{F}\left(\xi^{\curlyvee}\langle+,+\rangle\right)$ and $\mathscr{G}_{F}\left(\xi^{\curlyvee}\langle+,-\rangle\right)$. Assume the contrary. Let $r_{b} \in V$ be such that

$$
\mathscr{G}_{F^{\circ}}\left(\xi^{\frown}\langle+,-\rangle\right) \leq r_{b} \leq \mathscr{G}_{F^{\circ}}\left(\xi^{\urcorner}\langle+,+\rangle\right)
$$

By \#\#\#, this means that

$$
G_{F^{\circ}}\left(\xi^{\frown}\langle+\rangle\right)+\alpha^{\prime} \leq r_{b} \leq G_{F^{\circ}}\left(\xi^{\urcorner}\langle+\rangle\right)+\beta,
$$

contradicting \#\# (with $\alpha^{\prime}$ instead of $\alpha$ ). The case for $\langle-,+\rangle$ and $\langle-,-\rangle$ is similar. This completes the induction. 


\section{B. Proof of Fact 2}

For any higher-order gap model $M$ for SL and any predicate $F \in P_{S}$, for any $a \in E$, if $a \in \llbracket F \rrbracket$, then for any $b \in E$, if $\left|\mathscr{H}_{F}(a)-\mathscr{H}_{F}(b)\right| \leq \mathscr{T}(F)$, then $b \in \llbracket F \rrbracket$.

Proof. By straightforward induction over degrees of predicates (number of occurrences of $D$ ), from $\mathscr{T}$ and Definition 2 we can show that

$\left(^{*} \quad \quad\right.$ For any $k \geq 0$, if $F$ is a predicate of degree $k$, then

$$
\mathscr{T}(F)=\mathscr{G}_{F^{\circ}}\left(\xi^{\wedge}\langle+\rangle\right)-\mathscr{G}_{F^{\circ}}\left(\xi^{\wedge}\langle-\rangle\right),
$$

where $\xi \in T$ is any sequence of length $k$.

Further, it is immediate that

(**) If for any $a \in E$

$$
\left.a \in \llbracket F \rrbracket] \text { iff } \mathscr{H}_{F}(a)>\mathscr{G}_{F}\left(\xi^{\wedge}\langle+\rangle\right) \quad \text { and } \quad a \notin \llbracket F \rrbracket \text { iff } \mathscr{H}_{F}(a)<\mathscr{G}_{F}\left(\xi^{\wedge}\langle-\rangle\right)\right)
$$

then it also holds for any $a \in \llbracket F \rrbracket$ and any $b \in E$ that

$$
\text { if }\left|\mathscr{H}_{F^{\circ}}(a)-\mathscr{H}_{F^{\circ}}(b)\right| \leq \mathscr{G}_{F^{\circ}}\left(\xi^{\urcorner}\langle+\rangle\right)-\mathscr{G}_{F^{\circ}}\left(\xi^{\urcorner}\langle-\rangle\right), \quad \text { then } b \in \llbracket F \rrbracket
$$

Let's say that atomic predicates are positive; and if $F$ is positive [negative], then $\neg F$ is negative [positive]; and if $F$ is positive [negative], then $D F$ is positive [negative]. Let the degree of a predicate $F$ be the number of occurrences of $D$ in $F$. We show that the extensions of predicates follow the $T$-assignment in the following sense:

${ }^{(* *)}$ If $F$ is a positive [negative] predicate of degree $n \geq 0$, then there is a sequence $\xi^{\urcorner}\langle+\rangle \quad\left[\xi^{\curlyvee}\langle-\rangle\right]$, with length $l(\xi)=n$, such that for all $a \in E, a \in \llbracket F \rrbracket$ iff $\mathscr{H}_{F^{\circ}}(a)>$ $\mathscr{G}_{F^{\circ}}(\xi \prec\langle+\rangle) \quad\left[\mathscr{H}_{F^{\circ}}(a)<\mathscr{G}_{F^{\circ}}(\xi \prec\langle-\rangle)\right]$.

Showing this is is sufficient, for the assumption that $M$ is a higher-order gap model entails by definition that for any $\xi \in T$, and any $a \in E, \mathscr{H}_{F^{\circ}}(a)>G_{F^{\circ}}\left(\xi^{\urcorner}\langle+\rangle\right)$or $\mathscr{H}_{F}(a)<$ $G_{F}\left(\xi^{\wedge}\langle-\rangle\right)$. Then the Fact follows by $*$ and **: *** together with the assumption that

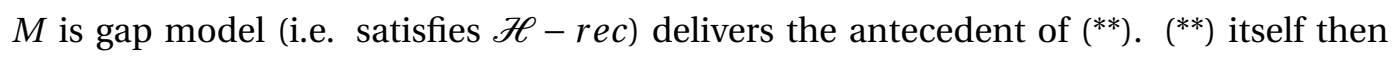
delivers the consequent of $* *$. Using (*), we substitute the left-hand side of $*$ for the right-hand side in the consequent of ** $^{*}$. We then have the Fact.

We prove ${ }^{* * *}$ by induction over predicate degree. It holds for atomic $F$ immediately by clause (ii) of SLS. For the induction step, assume that $F$ is a positive predicate of 
degree $k+1$. Then either $F$ is $D F^{\prime}$, where $F^{\prime}$ is a positive predicate, or $F$ is $\neg D F^{\prime}$, where $F^{\prime}$ is a negative predicate, of degree $k$. Assume the former. The other case is similar. By the induction hypothesis, there is a sequence $\xi^{\frown}\langle+\rangle$ such that $\llbracket F^{\prime} \rrbracket=\left\{a \in E: \mathscr{H}_{F^{\circ}}(a)\right\rangle$ $\left.\mathscr{G}_{F^{\circ}}(\xi \frown\langle+\rangle)\right\}$, where $l(\xi)=k$.

By clause (iv) of SLS, and the definitions $\mathscr{T}$ and $(\mathscr{S}), a \in \llbracket D F^{\prime} \rrbracket$ iff for any $b \in$ $E$, $\left|\mathscr{H}_{F^{\circ}}(a)-\mathscr{H}_{F^{\circ}}(b)\right| \leq \epsilon\left(\mathscr{T}\left(F^{\prime}\right)\right)$ it holds that $b \in \llbracket F^{\prime} \rrbracket$. By the induction hypothesis, if $b \in \llbracket F^{\prime} \rrbracket$, then $\left.\mathscr{H}_{F^{\circ}}(b)>\mathscr{G}_{F^{\circ}}(\xi\urcorner\langle+\rangle\right)$. Hence, if $a \in \llbracket D F^{\prime} \rrbracket$, then $\mathscr{H}_{F^{\circ}}(a)>\mathscr{G}_{F^{\circ}}(\xi \prec\langle+\rangle)+$ $\epsilon\left(\mathscr{T}\left(F^{\prime}\right)\right)$. By $\left.\left.\circledast\right), \mathscr{T}\left(F^{\prime}\right)=\mathscr{G}_{F^{\circ}}\left(\xi^{\urcorner}\langle+\rangle\right)-\mathscr{G}_{F^{\circ}}(\xi\urcorner\langle-\rangle\right)$. And by clause iiib) of Definition 2 , $\left.\left.\left.\mathscr{G}_{F^{\circ}}(\xi\urcorner\langle+,+\rangle\right)=\mathscr{G}_{F^{\circ}}\left(\xi^{\wedge}\langle+\rangle\right)+\epsilon\left(\mathscr{G}_{F^{\circ}}(\xi\urcorner\langle+\rangle\right)-\mathscr{G}_{F^{\circ}}(\xi\urcorner\langle-\rangle\right)\right)$. Therefore, if $a \in \llbracket D F^{\prime} \rrbracket$, then $\left.\mathscr{H}_{F^{\circ}}(a)>\mathscr{G}_{F^{\circ}}(\xi\urcorner\langle+,+\rangle\right)$, as desired.

Then consider a negative predicate of degree $k+1$. Assume that $F^{\prime}$ is positive, and consider $\neg D F^{\prime}$. By iii) of SLS, $a \in \llbracket \neg D F^{\prime} \rrbracket$ just in case $a \in E-\llbracket D F^{\prime} \rrbracket$. By the result for the positive case above, an object $b \in \llbracket D F^{\prime} \rrbracket$ iff for some sequence $\xi$ of length $k$, $\mathscr{H}_{F^{\circ}}(b)>\mathscr{G}_{F^{\circ}}\left(\xi^{\wedge}\langle+\rangle\right)$. Since $M$ is a higher-order gap model, there is no object $c \in E$ such that $\left.\left.G_{F^{\circ}}(\xi\urcorner\langle+\rangle\right) \geq \mathscr{H}_{F^{\circ}}(c) \geq G_{F^{\circ}}(\xi\urcorner\langle-\rangle\right)$. Therefore, $a \in \llbracket \neg D F^{\prime} \rrbracket$ iff $\mathscr{H}_{F}(a)<\mathscr{G}_{F}(\xi \prec\langle-\rangle)$, as desired. The other two cases ( $D F$ negative and $\neg D F$ positive) are similar. This completes the proof of ***).

\section{Proof of Fact 3}

(SLS) validates a) T, b) RN, c) B, and d) modus ponens.

Proof. a) The predicate counterpart of $\mathrm{T}$ is

$\left(\mathrm{T}_{\text {pred }}\right) \quad D F=F$

The validity is immediate from clause (iv) of (SLS), by clause ii) of Definition 2 and the definition $\mathscr{S}$ of the safety function $\mathscr{S}$. Since $\mathscr{S}(D F) \geq 0$, and hence $\mathscr{H}_{F^{\circ}}(a)-\mathscr{H}_{F^{\circ}}(a) \leq$ $\mathscr{S}(D F), a \in \llbracket F \rrbracket$ if $a \in \llbracket D F \rrbracket$.

b) The predicate counterpart of $\mathrm{RN}$ is

$\left(\mathrm{RN}_{\text {pred }}\right) \quad$ If $\left.\right|_{M} F$, then $\models_{M} D F$

It is valid. If $\mid=F_{M}, \llbracket F \rrbracket_{M}=E_{M}$. Then any $a \in E_{M}$ satisfies the condition for $\llbracket D F \rrbracket$, since it trivially holds for any $a \in E_{M}$ that $\forall b \in E_{M}\left(\left|\mathscr{H}_{F}(a)-\mathscr{H}_{F}(b)\right| \leq \mathscr{S}(D F)\right) \longrightarrow b \in \llbracket F \rrbracket$.

c) The predicate counterpart of $B$ is

(B pred $) \quad F=D \neg D \neg F$ 
(B $a \notin \llbracket D \neg D \neg F \rrbracket$. By clause (iv) of (SLS), and clause (b) of $(\mathscr{S})$, it then holds that

$$
\exists b \in E\left(\left|\mathscr{H}_{F^{\circ}}(a)-\mathscr{H}_{F^{\circ}}(b)\right|\right) \leq \mathscr{S}(D D F) \& b \notin \llbracket \neg D \neg F \rrbracket
$$

Let $b^{\prime}$ be such a $b$. By (iii) of SLS) we also have

$$
\left|\mathscr{H}_{F^{\circ}}(a)-\mathscr{H}_{F^{\circ}}\left(b^{\prime}\right)\right| \leq \mathscr{S}(D D F) \& b^{\prime} \in \llbracket D \neg F \rrbracket
$$

Let $\left|\mathscr{H}_{F^{\circ}}(a)-\mathscr{H}_{F^{\circ}}\left(b^{\prime}\right)\right|=j$, where $0 \leq j \leq \mathscr{S}(D D F)$. This means that either $\mathscr{H}_{F^{\circ}}(a)-$ $\mathscr{H}_{F^{\circ}}\left(b^{\prime}\right)=j$, or $\mathscr{H}_{F^{\circ}}(a)-\mathscr{H}_{F^{\circ}}\left(b^{\prime}\right)=-j$.

Again, by clause (iv) of (SLS), it holds that

$$
\left.\forall c \in E\left(\left|\mathscr{H}_{F^{\circ}}\left(b^{\prime}\right)-\mathscr{H}_{F^{\circ}}(c)\right|\right) \leq \mathscr{S}(D F) \rightarrow c \in \llbracket \neg F \rrbracket\right)
$$

Since $\mathscr{H}_{F^{\circ}}(a)-\mathscr{H}_{F^{\circ}}(c)=\mathscr{H}_{F^{\circ}}(a)-\mathscr{H}_{F^{\circ}}\left(b^{\prime}\right)+\mathscr{H}_{F^{\circ}}\left(b^{\prime}\right)-\mathscr{H}_{F^{\circ}}(c)$, it holds either that $\left|\mathscr{H}_{F^{\circ}}(a)-\mathscr{H}_{F^{\circ}}(c)\right|=\left|\mathscr{H}_{F^{\circ}}\left(b^{\prime}\right)-\mathscr{H}_{F^{\circ}}(c)\right|+j$, or else that $\left|\mathscr{H}_{F^{\circ}}(a)-\mathscr{H}_{F^{\circ}}(c)\right|=\mid \mathscr{H}_{F^{\circ}}\left(b^{\prime}\right)-$ $\mathscr{H}_{F^{\circ}}(c) \mid-j$

It therefore follows from $(\$$ ) and $(\$ \$$ ) that

$$
\begin{aligned}
& \forall c \in E\left(\left|\mathscr{H}_{F^{\circ}}(a)-\mathscr{H}_{F^{\circ}}(c)\right| \leq \mathscr{S}(D F)+j \rightarrow c \notin \llbracket F \rrbracket\right), \text { or } \\
& \forall c \in E\left(\left|\mathscr{H}_{F^{\circ}}(a)-\mathscr{H}_{F^{\circ}}(c)\right| \leq \mathscr{S}(D F)-j \rightarrow c \notin \llbracket F \rrbracket\right)
\end{aligned}
$$

Since $\mathscr{S}(G) \geq 0$, for any $G$, it holds that $\left|\mathscr{H}_{F^{\circ}}(a)-\mathscr{H}_{F^{\circ}}(a)\right| \leq j+\mathscr{S}(D F)$. Further, by the definitions of $\mathscr{S}$ and $\mathscr{T}$, and by clauses ib) and iib) of Definition 2 it holds that $\mathscr{S}(D D F) \leq \mathscr{S}(D F)$, and hence that $j \leq \mathscr{S}(D F)$. Therefore, it also holds that $\mid \mathscr{H}_{F^{\circ}}(a)-$ $\mathscr{H}_{F^{\circ}}(a) \mid \leq \mathscr{S}(D F)-j$. Either way, it then follows from $\$ \$ \S$ that $a \notin \llbracket F \rrbracket$. Hence, if $a \notin \llbracket D \neg D \neg F \rrbracket$, then $a \notin \llbracket F \rrbracket$, which establishes c).

d) The tolerance logic counterpart of modus ponens is valid:

$\left(\mathrm{MP}_{\text {pred }}\right) \quad$ From $F \vDash G$ and $\models F$, infer $\vDash G$

Immediate from (STL): if, by (ii) and (i), $\llbracket F \rrbracket_{M} \subseteq \llbracket G \rrbracket_{M}$ and $\llbracket F \rrbracket_{M}=E_{M}$, then $\llbracket G \rrbracket_{M}=E_{M}$.

\section{Proof of Fact 4}

a): $\quad F\left|=_{M} G \Rightarrow D F\right|{ }_{M} D G$

b): $F=G \quad \Rightarrow \quad D F \mid=D G$

Proof. Proof of a). By means of a simple countermodel. Consider a set of men and the 
predicate 'tall' $T$. The central gap has size $1 \mathrm{~cm}$ and the upper edge at $180 \mathrm{~cm}$. The $\delta$ and $\epsilon$ function are such that $\delta(x)=0.9 x$ and $\epsilon(x)=10 x$.

Then the upper boundary for being $\neg T$ is at $179 \mathrm{~cm}$. The upper boundary for being $D \neg T$ is $179-10 \times 1=169 \mathrm{~cm}$. The lower boundary for being $\neg D \neg T$ is $169+0,9 \times 1=$ $169.9 \mathrm{~cm}$. The lower boundary for being $D \neg D \neg T$ is $169.9+10 \times 0.9=178.9 \mathrm{~cm}$. The lower boundary for being $D D \neg D \neg T$ is then $178.9+10 \times 0.9 \times 0.9=178.9+8.1=187 \mathrm{~cm}$. Repeating this, we find that the lower boundary for being $D^{6} \neg D \neg T$ is greater than the lower boundary for being $D^{4} T$, but that the result of prefixing both with $D$ yields the reverse result: the lower boundary of being $D^{7} \neg D \neg T$ is slightly lower than the lower boundary of being $D^{5} T$. Hence, we have $D^{6} \neg D \neg T \vDash_{M} D^{4} T$, but $D^{7} \neg D \neg T \not \vDash_{M} D^{5} T$.

Proof of b). By means of counterexample. Let $F$ be $\neg D \neg D G$. We can show that $F \vDash G$, by a reasoning that parallels the proof that (SLS) validates B. Here, I shall simplify the argument. Assume without loss of generality that $G$ is a positive predicate (elements of its extension have higher measure than elements of its anti-extension). Let $\gamma$ be $\mathscr{T}(G)$, and let $\beta$ be the upper boundary of the $G$ gap. We must now show that $\llbracket F \rrbracket \subseteq \llbracket G \rrbracket$, which means if an object $a$ is in $\llbracket F \rrbracket$, then its measure is not below the $G$ gap. That is: $\mathscr{H}_{G^{\circ}}(a) \geq$ $\beta-\gamma$.

The upper boundary $F^{+}$of the gap for $F$ is, by Definition 2 , calculated as:

(о) $\quad F^{+}=\beta+\epsilon(\gamma)-\delta(\gamma)-\epsilon(\delta(\gamma))+\delta(\delta(\gamma))$.

Since no element is in the $G$ gap, any object above the lower boundary of the $G$ gap in in $G$. So what we need is that $F^{+}>\beta-\gamma$. That is

(००) $\quad \beta+\epsilon(\gamma)-\delta(\gamma)-\epsilon(\delta(\gamma))+\delta(\delta(\gamma))>\beta-\gamma$

Simplifying, this holds iff

$(\circ \circ \circ) \quad \gamma+\epsilon(\gamma)-\delta(\gamma)-\epsilon(\delta(\gamma))+\delta(\delta(\gamma))>0$

Since, by Definition 2, a) it holds that $\gamma \geq \delta(\gamma)$, b) it holds that $\epsilon(\gamma) \geq \epsilon(\delta(\gamma))$, and c) it holds that $\delta(\delta(\gamma))>0$, $\circ \circ \circ$ is true. Hence, $\llbracket F \rrbracket \subseteq \llbracket G \rrbracket$.

Nevertheless, by suitable choices of $\epsilon$ and $\delta$, we can have a model, along the lines of the proof of part a) of the Fact, where $\llbracket D F \rrbracket \nsubseteq \llbracket D G \rrbracket$

\section{References}

Bobzien, Susanne (Aug. 2010). "Higher-order Vagueness, Radical Unclarity, and Absolute Agnosticism”. In: Philosopher's Imprint 10, pp. 1-30. 
Bobzien, Susanne (2013). "Higher-order Vagueness and Borderline Nestings: A Persistent Confusion”. In: Analytic Philosophy 54, pp. 1-43.

Cobreros, Pablo (2011a). "Paraconsistent Vagueness: A Positive Argument”. In: Synthese 183, pp. 211-27.

- (2011b). "Varzi on Supervaluationism and Logical Consequence”. In: Mind 120, pp. 83343.

Dietz, Richard (2015). "The Myth of Rdical Higher-order Vagueness". Unpublished.

Dietz, Richard and Sebastiano Moruzzi, eds. (2010). Cuts and Clouds. Oxford: Oxford University Press.

Eklund, Matti (2007). “Characterizing Vagueness”. In: Philosophy Compass 2, pp. 896909.

Fara, Delia Graff (2002). “An Anti-Epistemicist Consequence of Margin for Error Semantics for Knowledge”. In: Philosophy and Phenomenological Research 64, pp. 127-42.

- (2003). "Gap Principles, Penumbral Consequence, and Infinitely Higher-Order Vagueness”. In: Liars and Heaps. Ed. by J. C. Beall. Oxford: Oxford University Press, pp. 195221.

Fine, Kit (1975). "Vagueness, Truth and Logic”. In: Synthese 30, pp. 265-300.

- (2008). “The Impossibility of Vagueness”. In: Philosophical Perspectives 22, pp. 11136.

Keefe, Rosanna (2000). Theories of Vagueness. Cambridge: Cambridge University Press.

Keefe, Rosanna and Peter Smith (1996a). “Introduction: theories of vagueness”. In: Vagueness: A Reader. Ed. by Rosanna Keefe and Peter Smith. MIT Press, pp. 1-57.

- eds. (1996b). Vagueness: A Reader. Cambridege, MA: MIT Press.

Mahtani, Anni (2008). "Can Vagueness Cut Out at Any Order”. In: Australasian Journal of Philosophy 86, pp. 499-508.

Negri, Sara (2011). “Proof Theory for Modal Logic”. In: Philosophy Compass 6, pp. 523-38.

Pagin, Peter (2010). "Vagueness and Central Gaps". In: Cuts and Clouds. Ed. by Richard Dietz and Sebastiano Moruzzi. Oxford: Oxford University Press, pp. 254-72.

- (2011a). “Information and Assertoric Force”. In: Assertion. Ed. by Jessica Brown and Herman Cappelen. Oxford: Oxford University Press, pp. 97-136.

- (2011b). "Vagueness and Domain Restriction". In: Vagueness and Language Use. Ed. by Paul Egré and Nathan Klinedinst. Basingstoke: Palgrave MacMillan, pp. 283-307.

Raffman, Diana (2010). “Demoting Higher-order Vagueness”. In: Cuts and Clouds. Ed. by Richard Dietz and Sebastiano Moruzzi. Oxford: Oxford University Press, pp. 509-22. 
Russell, Bertrand (1923). "Vagueness". In: Australasian Journal of Philosophy and Psychology 1, pp. 84-92. Reprinted in Keefe and Smith 1996b, 61-8. Page references to the reprint.

Sainsbury, R. M. (1990). “Concepts without Boundaries”. In: Vagueness. A Reader. Ed. by Rosanna Keefe and Peter Smith. MIT Press, pp. 251-64. Inaugural lecture delivered 6 November 1990. Book published 1996.

— (1991). "Is There Higher-order Vagueness?" In: The Philosophical Quarterly 41.163, pp. 167-82.

Schiffer, Stephen (2003). The Things We Mean. Oxford: Oxford University Press.

Sorensen, Roy (1988). Blindspots. Oxford: Clarendon Press.

Westerståhl, Dag (1985). “Determiners and Context Sets”. In: Generalized Quantifiers in Natural Language. Ed. by Johan van Benthem and Alice ter Meulen. Amsterdam: Foris, pp. 45-71.

Williamson, Timothy (1994). Vagueness. London: Routledge.

- (1999). “On the Structure of Higher-Order Vagueness”. In: Mind 108.429, pp. 127-143.

- (2000). Knowledge and its Limits. Oxford: Oxford University Press.

Wright, Crispin (1975). “On the Coherence of Vague Predicates”. In: Synthese 30, pp. 325365.

- (1976). "Language-mastery and the sorites paradox". In: Truth and Meaning. Ed. by Gareth Evans and John McDowell. Oxford: Clarendon Press, pp. 223-47.

- (2010). “The Illusion of Higher-order Vagueness". In: Cuts and Clouds. Ed. by Richard Dietz and Sebastiano Moruzzi. Oxford: Oxford University Press, pp. 523-49.

Zardini, Elia (2013). “Higher-order Sorites Paradox”. In: Journal of Philosophical Logic 42, pp. 25-48. 